\title{
Research Paper \\ Effect of Donepezil and Hyoscyamoside on Improving Spatial Memory in Rats With Alzheimer's Disease
}

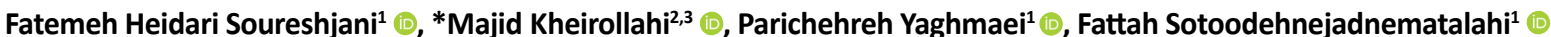

1. Department of Biology, Faculty of Basic Sciences, Science and Research Branch, Islamic Azad University, Tehran, Iran.

2. Department of Genetics and Molecular Biology, School of Medicine, Isfahan University of Medical Sciences, Isfahan, Iran.

3. Pediatric Inherited Diseases Research Center, Research Institute for Primordial Prevention of Non-communicable Disease, Isfahan University of Medical Sciences, Isfahan, Iran.

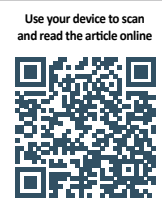

Cittation: Heidari Soureshjani F, Kheirollahi M, Yaghmaei P, Sotoodehnejadnematalahi F. [Effect of donepezil and hyoscyamoside on improving spatial memory in rats With Alzheimer's Disease (Persian)]. Journal of Arak University of Medical Sciences(JAMS). 2020; 23(4):524-539. https://doi.org/10.32598/JAMS.23.4.6135.1

doi hittps://doi.org/10.32598/JAMS.23.4.6135.1

Keywords:

Alzheimer's disease, Hyoscyamoside, Donepezil, Morris water maze, Spatial memory

\section{AB STRACT}

Background and Aim Alzheimer's Disease (AD) is a neurodegenerative brain disease that gradually destroys memory and cognitive skills. The disease is caused by the formation of beta-amyloid plaques, oxidative stress, dysfunctions in the cholinergic system, neuronal killing inflammation, and ultimately brain atrophy. Donepezil and hyoscyamoside have inhibitory effects on these pathogens; therefore, their impact on the learning process of Alzheimer's rats in the Morris Water Maze was investigated.

Methods \& Materials In the present experimental study, 60 male rats of Wistar breed with approximately 7 weeks age within the control group (rats that received normal water and food), the PBS group (underwent surgery), PBS group (received solvent A $\beta$ ), the first Alzheimerss group (animals that received betaamyloid by Alzheimer's surgery, second Alzheimer's group (after Alzheimer's surgery, they received $1 \mathrm{cc}$ of normal saline daily, and treatment groups that treated the rats with beta-amyloid after Alzheimer. In the hyoscyamoside group, they received $10 \mathrm{mg} / \mathrm{kg}$ daily of hyoscyamoside for 28 days. The donepezil group received it $4 \mathrm{mg} / \mathrm{kg}$ daily for 28 days by gavage. The Morris Water Maze test was used to evaluate learning and memory. Data were analyzed by ANOVA statistical analysis and Post Hoc test.

Ethical Considerations The Ethics Committee in Biomedical Research, Islamic Azad University, Science and Research Branch approved the research (Code: IR.IAU.SRB.REC 1397.057)

Results Beta-amyloid injection caused extensive damage to memory. The treatment groups with hyoscyamoside and donepezil spent less time and distance with a significant level $(P<0.001)$ than the group of Alzheimer's patients to find the hidden platform. In the reminder phase, where the previously hidden platform was located, they spent more time, with a significant level $(P<0.001)$ in the local quarter. Conclusion Treatment of rats with hyoscyamoside and donepezil improved spatial memory in Alzheimer's rats. They appear to play a significant role in the prevention and treatment of Alzheimer's disease

\section{Extended Abstract}

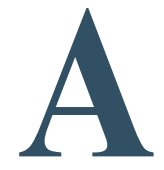

\section{Introduction}

lzheimer's Disease (AD) is a neurodegenerative disease that is associated with pro- found impairments of memory and cognitive functions. In this disease, amyloid accumulation with hyperphosphorylation of tau protein, creating oxidative stress, inflammation, altered secretion of mediators, inhibition of synaptic plasticity, decreased number and function of cholinergic neurons, and eventually cell death. Research has shown that the use

Corresponding Author:

Majid Kheirollahi, PhD.

Address: Department of Genetics and Molecular Biology, School of Medicine, Isfahan University of Medical Sciences, Isfahan, Iran.

Tel: +98 (31) 37929086

E-mail: mkheirollahi@med.mui.ac.ir 
of steroid saponins reduces pro-inflammatory factors in the brain. Other benefits include their antioxidant and anti-acetylcholinesterase properties [5].

The use of medicinal plants with low side effects and antioxidant properties has received much attention. Therefore, we investigated the effect of sapogenin (hyoscyamoside) on spatial memory, and perhaps it can be used as a dietary supplement for families with hereditary Alzheimer's disease in the prevention, delay of pathology, and treatment of this disease.

\section{Materials and Methods}

In the present experimental study, 60 male Wistar rats, approximately 7 weeks old, were divided into the following groups: control group, consisted of rats that received normal water and food; PBS group underwent surgery and received PBS (A $\beta$ solvent); the first Alzheimer's group consisted of rats that received beta amyloid during Alzheimer's surgery; the second Alzheimer's group received $1 \mathrm{cc}$ of normal saline daily after Alzheimer's surgery; the treatment groups, after beta-amyloid induction of Alzheimer's disease in the rats, received $10 \mathrm{mg} / \mathrm{kg}$ of hyoscyamoside for 28 days in the hyoscyamoside group; the donepezil group received $4 \mathrm{mg} / \mathrm{kg}$ of this substance by gavage for 28 days.

Morris Water Maze (MWM) test was used to assess learning and memory. Due to the lack of differences in the initial results of the MWM test in the control group and PBS group, the control group was considered as the control group and therefore the second Alzheimer's group was considered as the Alzheimer's group. In this study, SPSS software V. 22 was used. To investigate the existence of significant differences between the groups, Analysis of Variance (ANOVA) and post hoc tests were used according to the data.

\section{Results}

MWM test was used to evaluate spatial learning and memory. The results showed that the learning and spatial memory of the rats that received $A \beta$ as an intra-hippocampal injection
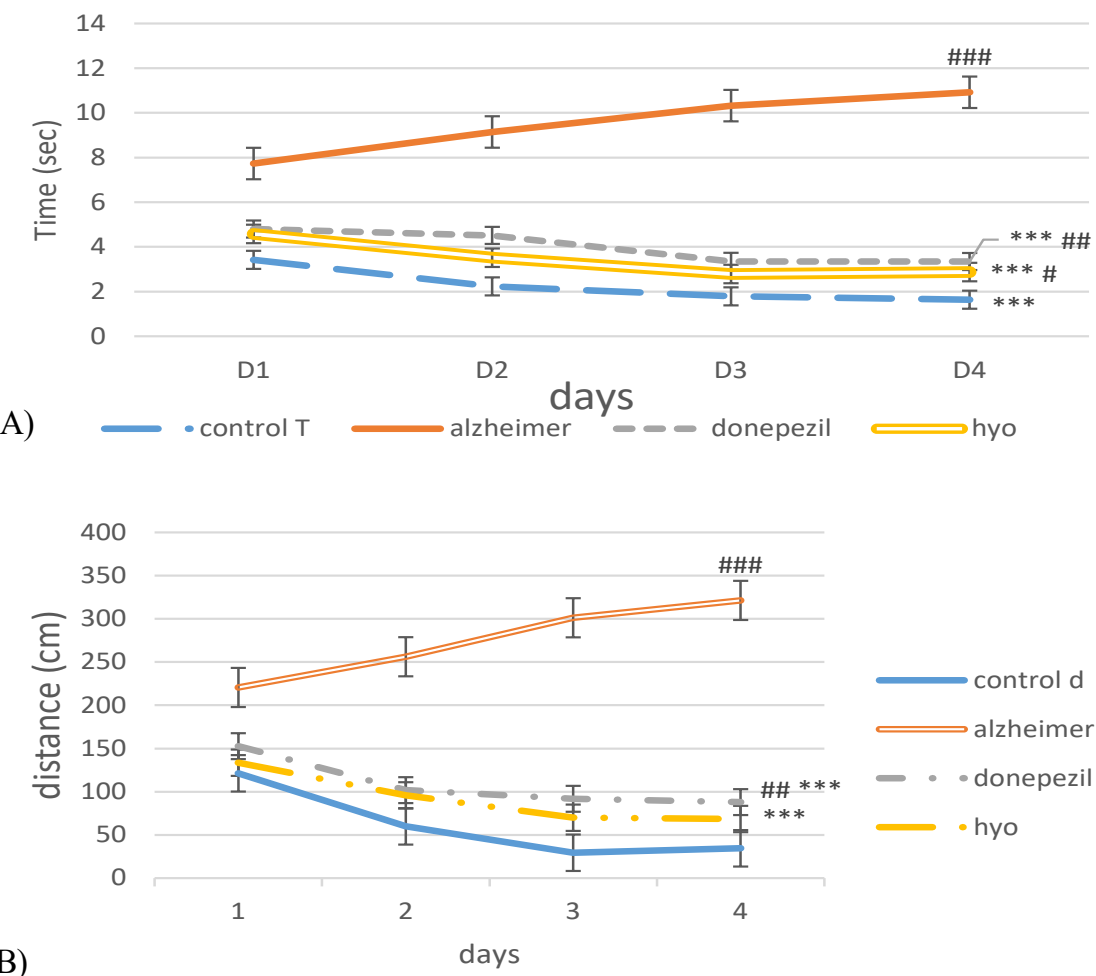

Journal of
Arak University of Medical Sciences

Figure 1. Effect of treatment with donepezil and Hyoscyamoside (Hyo) on Alzheimer's animals using the Morris Water navigation task

A: Elapsed time; B: The distance swam in the Morris water navigation task to find the hidden platform on different days of the experiment;

$* * \mathrm{P}<0.01 ; * * * \mathrm{P}<0.001$, Significant difference compared to Alzheimer's group;

${ }^{\#} \mathrm{P}<0.01 ;{ }^{\# \#} \mathrm{P}<0.001$, Significant difference compared to the control group. 


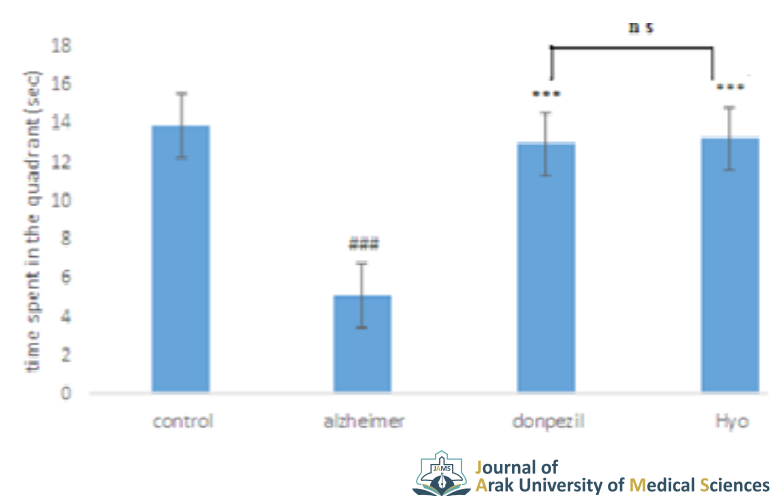

Figure 2. The effect of treatments on Alzheimer's rats in the dot-probe test in the Morris water navigation task

***P<0/001, Significant difference compared to Alzheimer's group;

${ }^{\#} \mathrm{P}<0 / 001$, Significant difference compared to the control group;

NS: Nonsignificant.

were impaired $(\mathrm{P}<0.001)$. The time elapsed and the distance traveled to reach the hidden platform, in the first to third days of training were considered as the criteria for learning, and on the fourth day after learning, they were considered as the criteria for verification. Alzheimer's rats spent significantly more time and distance to find the hidden platform than the control group $(\mathrm{P}<0.001)$.

The treatment groups showed a significant decrease $(\mathrm{P}<0.001)$ compared to the Alzheimer's group. On the fourth day, the hyoscyamoside group showed a significant difference $(\mathrm{P}<0.05)$ in the elapsed time compared to the control group. The donepezil treatment group showed a significant difference $(\mathrm{P}<0.05)$ compared to the hyoscyamoside group. Regarding the distance traveled, the donepezil group showed a significant difference $(\mathrm{P}<0.01)$ compared to the control group, while the hyoscyamoside group had a significant difference $(\mathrm{P}<0.01)$ compared to the control group. In the treatment groups, significant improvement in learning disability and spatial memory of $\mathrm{A} \beta$-receiving rats were observed $(\mathrm{P}<0.001)$. The elapsed time and distance traveled to reach the hidden platform in the rats in the treatment groups decreased compared to the Alzheimer's group (Figure 1).

In the dot-probe test, Figure 2 showed a significant increase in the time spent in the target quadrant by the treatment groups compared to the Alzheimer's group $(\mathrm{P}<0.001)$, and this showed the positive effect of treatments on learning and spatial memory of Alzheimer's rats. The treatment groups were not significantly different from each other and the control group $(\mathrm{P}<0.05)$.

\section{Discussion and Conclusion}

In this study, Alzheimer's disease in rats reduced their spatial memory, and their spatial memory improved after treatments. The time and the distance to reach the podium in the rats' treatment groups were significantly reduced compared to the Alzheimer's group (Figure 1). Studies showed that induction of Alzheimer's disease in rats by $A \beta 1-42$, reduced their memory, and triggered an inflammatory response. Treatment with donepezil improved Alzheimer's rats' performance on the Morris water navigation task and reduced their inflammatory cytokines [16].

The use of the steroidal saponin diosgenin in mutant rats improved their memory and showed that the diosgenin treatment group removed $\mathrm{A} \beta$ plaque, reduced neuronal death and neurofibrillary tangles in the cerebral cortex and hippocampus, and inhibited acetylcholinesterase [15]. Sapogenins with antioxidant properties inhibit IL-6 and TNF $\alpha$ and inhibit their production of free radicals [27].

Researchers are trying to develop drugs that, in addition to having better effects, also have fewer side effects. Plants are one of the sources that have always been used for the production of new medicines [17]. The present results showed that the use of donepezil and hyoscyamoside improved memory. The treatments may have improved the condition of plaque formation and memory loss (due to plaque) and cholinergic system defects, which may be due to the inhibitory activity of acetylcholinesterase and antioxidants.

\section{Conclusion}

According to the results of studies in people at high risk of Alzheimer's disease, it is suggested that the use of hyoscyamoside and donepezil be included in their daily diet for prevention. In people with Alzheimer's, taking hyoscyamoside may improve the disease, possibly by influencing the causes of Alzheimer's (such as accumulated beta-amyloid plaque, cholinergic system disorders, oxidative stress, and inflammation), and prevention of neuronal apoptosis improves the disease and prevents familial cases of Alzheimer's.

\section{Ethical Considerations}

\section{Compliance with ethical guidelines}

This research was ethically approved by the Islamic Azad University, Science and Research Branch (Code: REC.1397.057. IR.IAU.SRB). 
Funding

The paper was extracted from the $\mathrm{PhD}$. dissertation of the first author, Department of Biology, Islamic Azad University, Science and Research Branch, Tehran.

Authors' contributions

All authors met the standard writing criteria based on the recommendations of the International Committee of Medical Journal Publishers (ICMJE) and all contributed equally to the writing of the work.

\section{Conflicts of interest}

The authors declared no conflict of interest.

\section{Acknowledgements}

This article was taken from a doctoral dissertation and has no sponsorship. We are grateful to all those who cooperated with us in conducting this research. 
This Page Intentionally Left Blank 


\title{
اثر دونيزيل و هيوسياموزيد بر بهبود حافظه فضايى در رتهاى مبتلا به آلزايمر
}

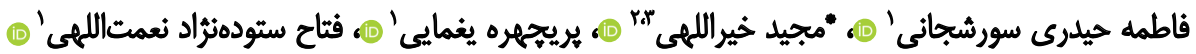

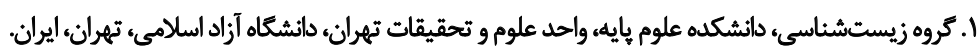

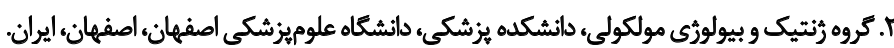

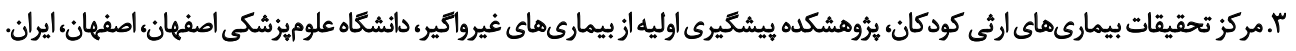

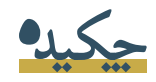

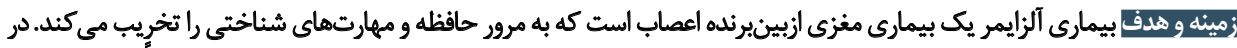

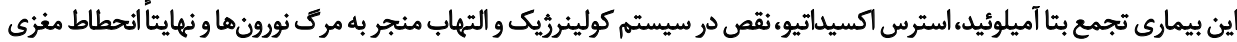

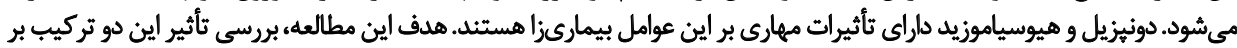
حافظه و يادكيرى موش هائ صحرايى آلزايمرى بودي

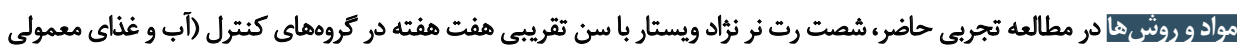

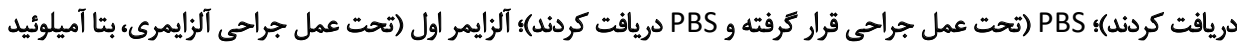

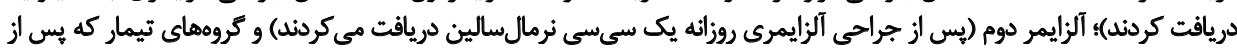

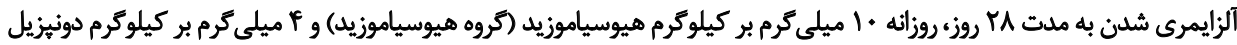

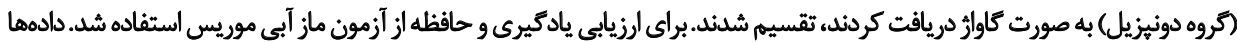

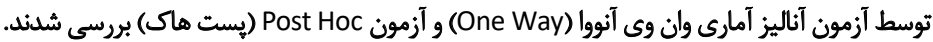

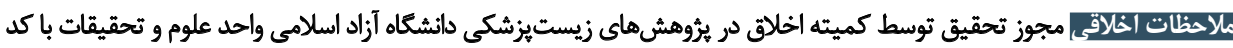
IR.IAU.SRB.REC 1397.057

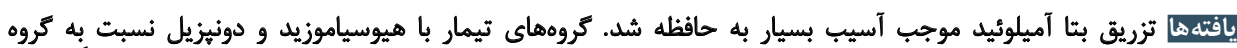

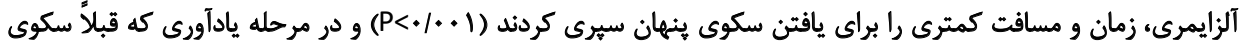

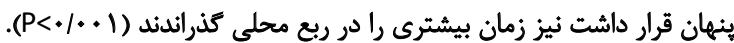

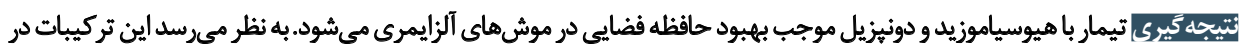

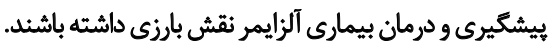

اطلاعات مقاله:

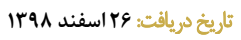

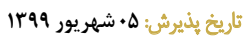

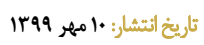

كليدوازوها:

بيمارى آلزايمر، هيوسياموزيد، دونيزيلة آزمون ماز آبي موريس، حافظه فضايي مازي

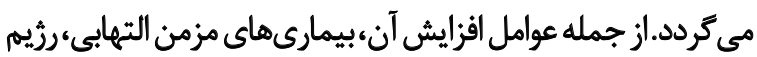

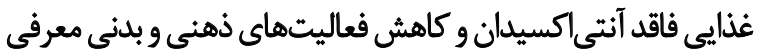

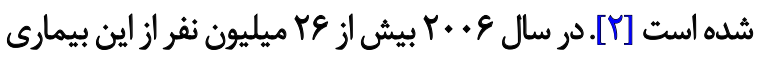

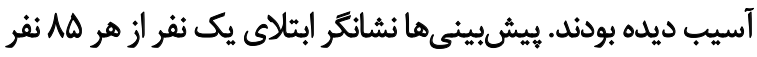

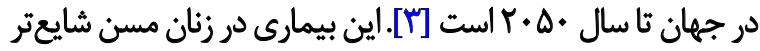

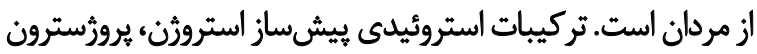

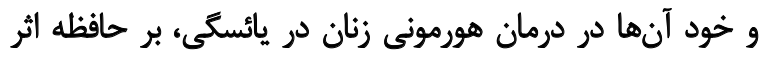

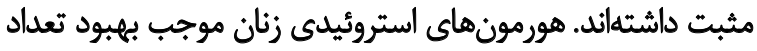

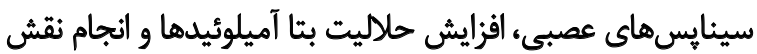

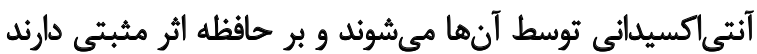

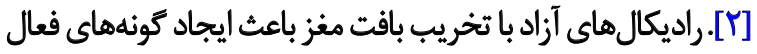

doles

بيمارى آلزايمر ' يك بيمارى نورودرثراتيو است كه بـا اختلالات

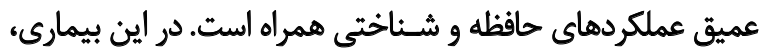

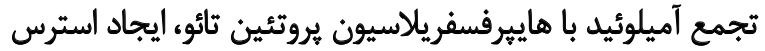

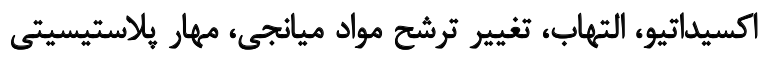

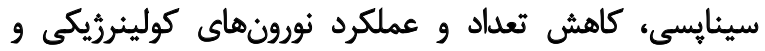

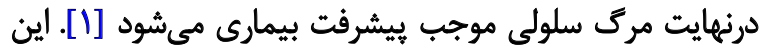

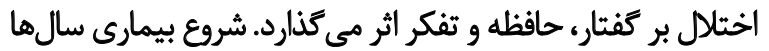

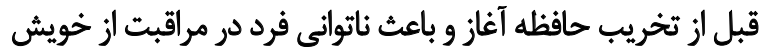

1. Alzheimer's Disease (AD)

\section{-}

"نويسنده مسئول:

كتر مجيد خير اللإنى

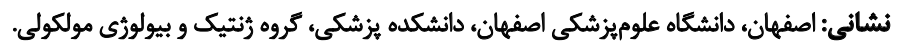

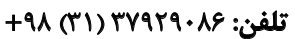
mkheirollahi@med.mui.ac.ir يست الكترونيكي 
سايونينها حمايت مى كنيند. استفاده از سايورنين استروئيدى باعث بهبود حافظه در مدل موش

بتا آميلوئيد شد [9]د

دونيزيل يك مهاركننده غيررقابتى وبازگتشتيذير استيل كولين

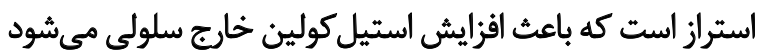

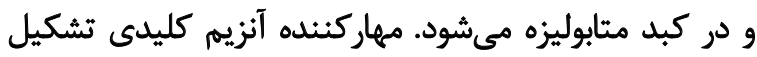

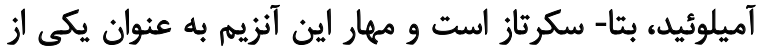

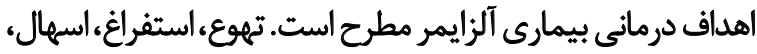

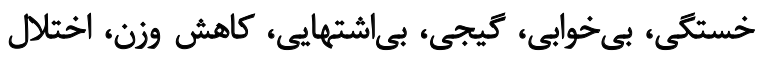

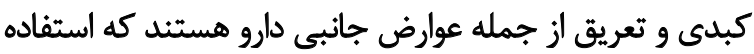

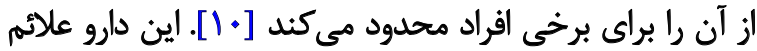

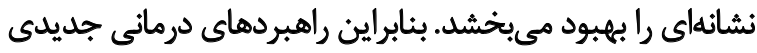

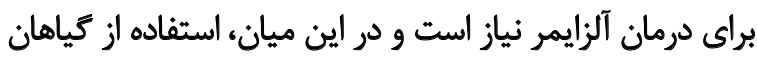

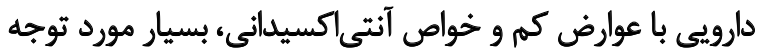

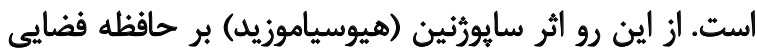

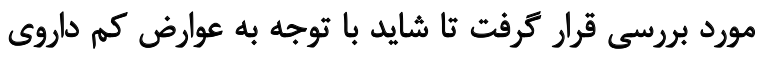

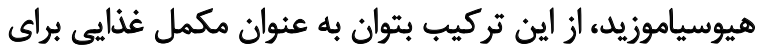

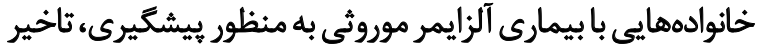
ياتولورثى و درمان بيمارى استفاده كردا.

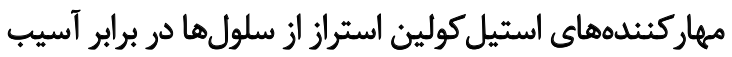

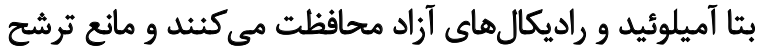

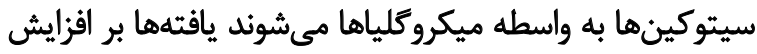

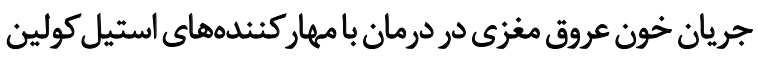

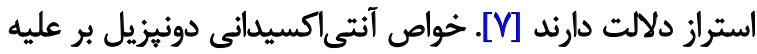

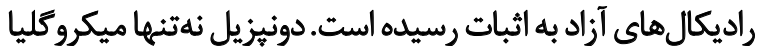

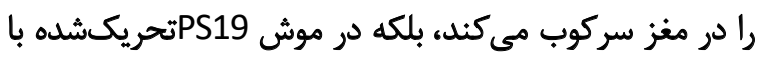

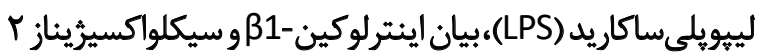

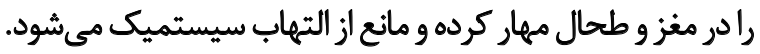

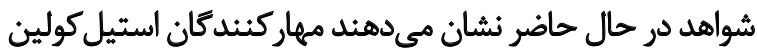

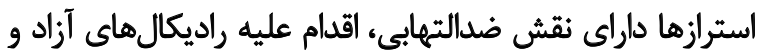
سميت آميلوئيد از طريق كاهش انتشار سيتوكينها التهاز ميكروكليا

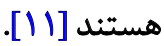

التهاب ناشى از يلاكهاى آميلوئيدى در بيماران آلزايمرى از

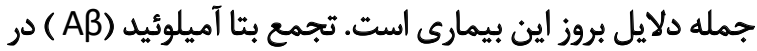

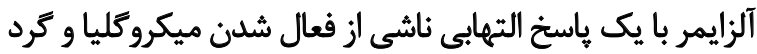

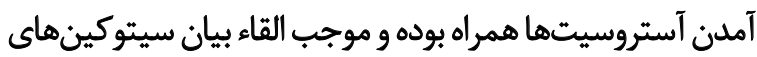

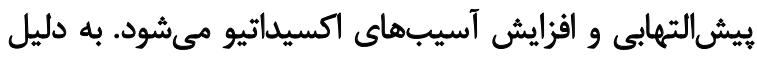

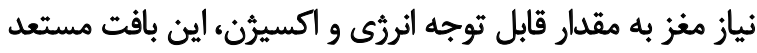

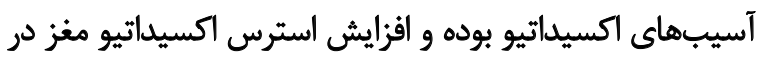

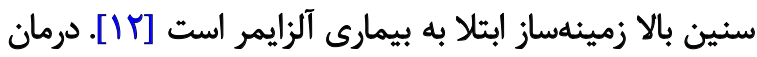

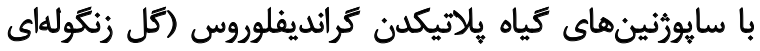

اكسيرن ب مىشوند و عمل ميانجىهاى نورونى را مختل مى كنيند، درنتيجه از عوامل اصلى در آلزايمر و تخريب نورئ نورونى هستئند

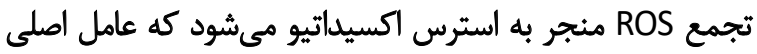

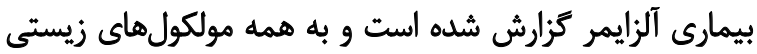

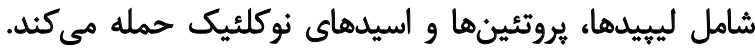

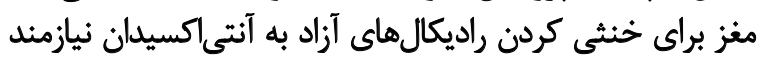

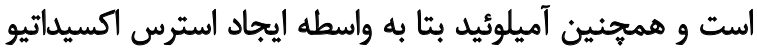

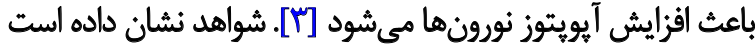
كه رزيم حاوى مواد غذايى مديترانهاي (سرشار ازئ آنتىاكسيداني)

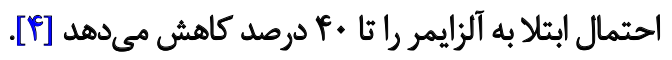
كياهان دارويى در سالهاى اخير به دليل داشتن اثرات متنوع،

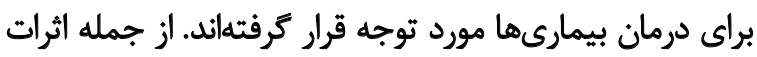

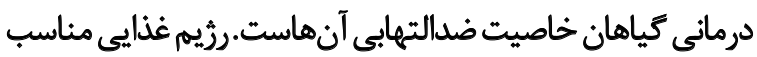

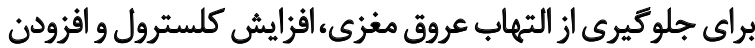

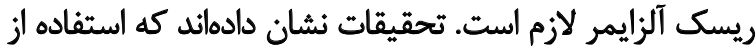

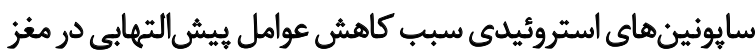

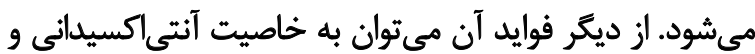

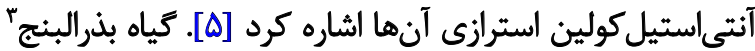

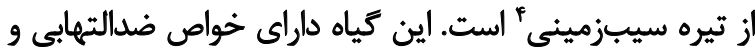

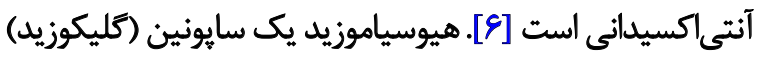

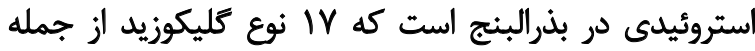

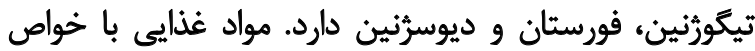

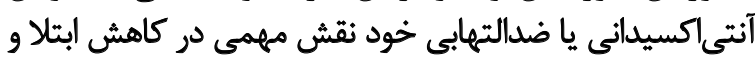

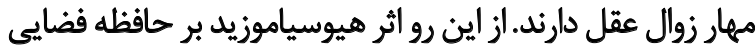

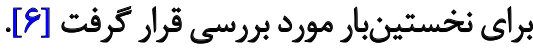

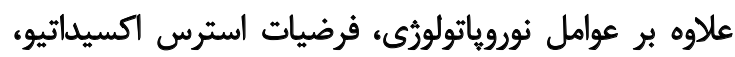

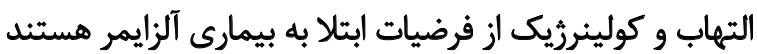

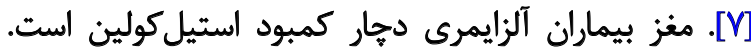

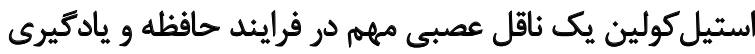

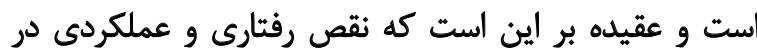

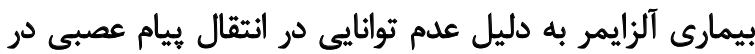

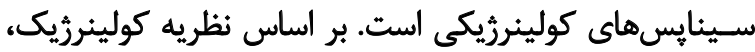

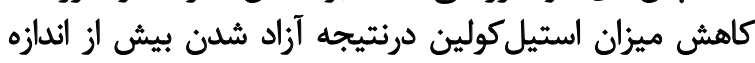

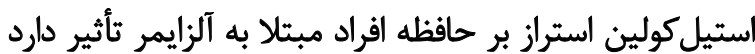

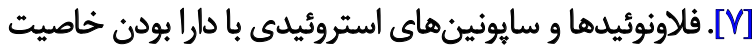

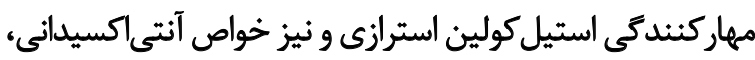

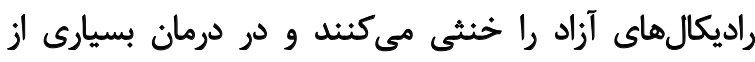

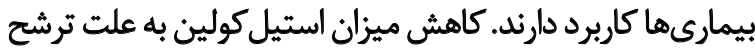

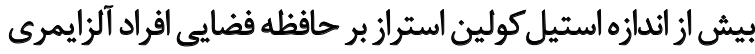

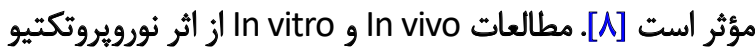

2. Reactive Oxygen Species (ROS)

3. Hyoscyamus niger

4. Solanaceae 
AKT/ سايورئين بر كاهش آيويتوز را به نقش آن بر تقويت مسين PI3K

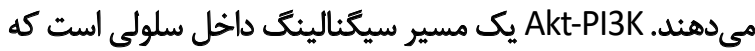

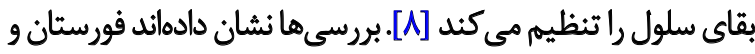

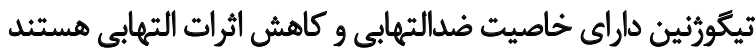

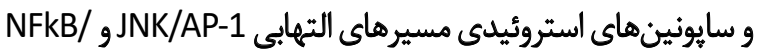

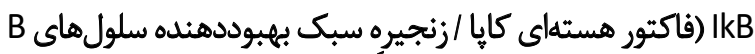

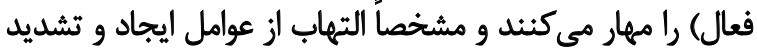

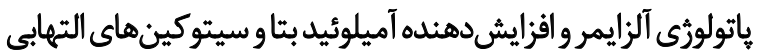

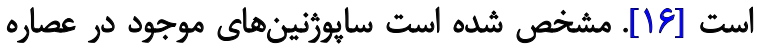

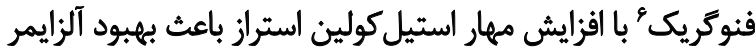

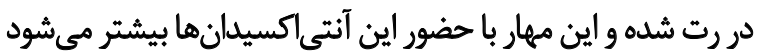

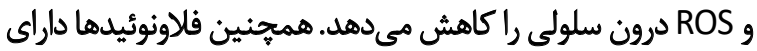

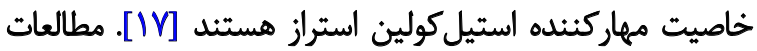

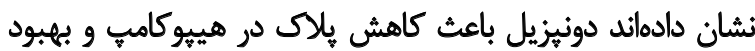

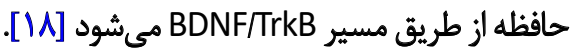

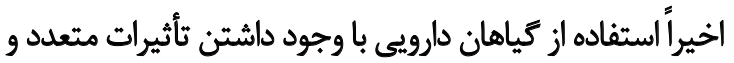

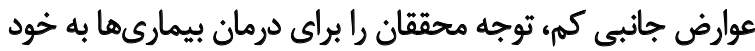

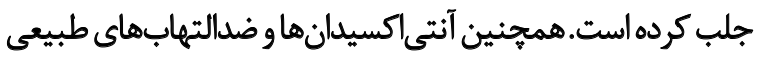

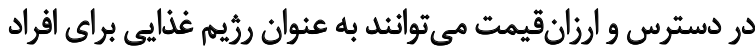

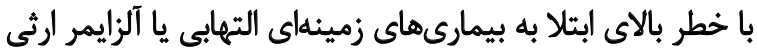

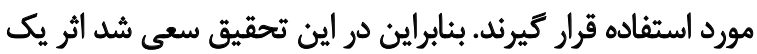

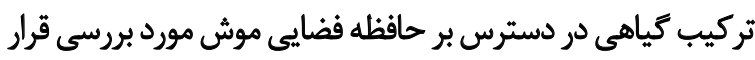

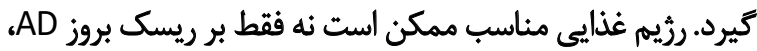

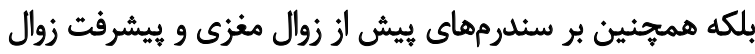

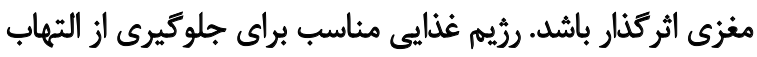

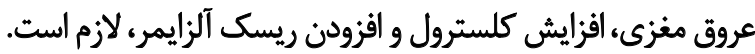

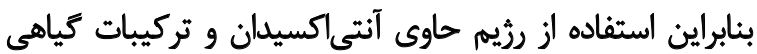

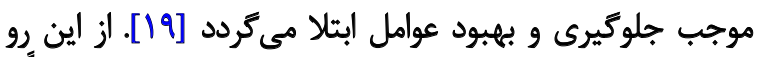

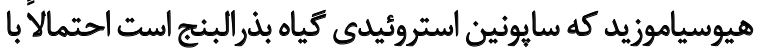

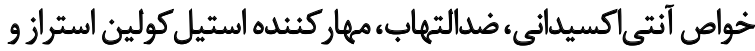

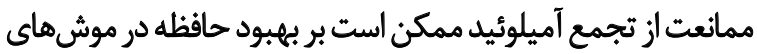

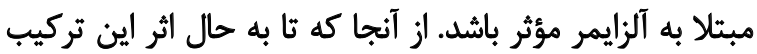

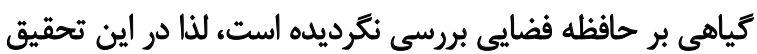

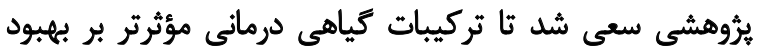

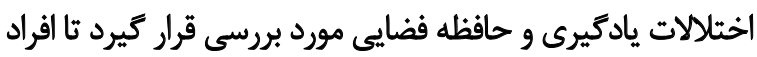

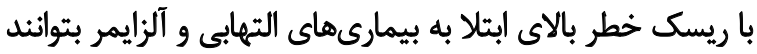

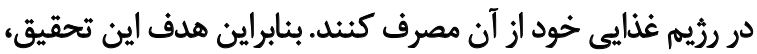

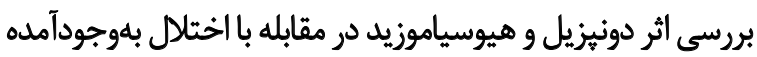

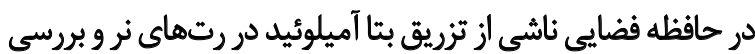

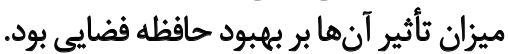

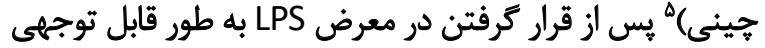

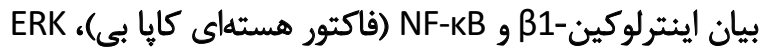

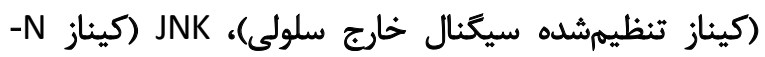

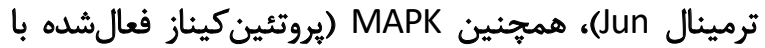

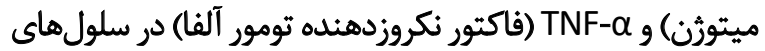

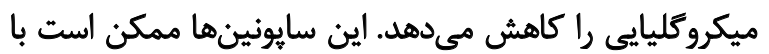

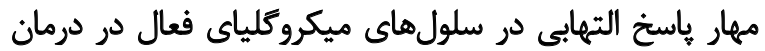

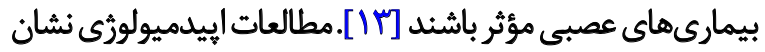

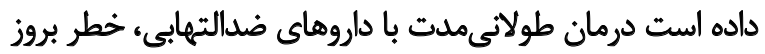

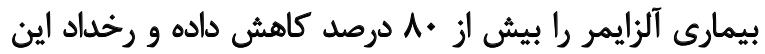

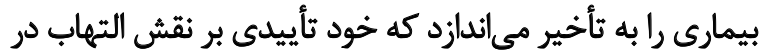

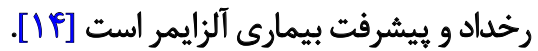

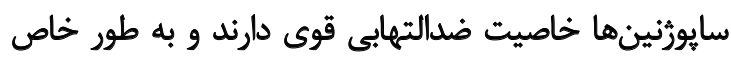

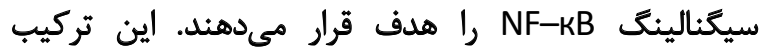

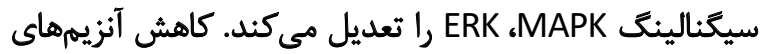

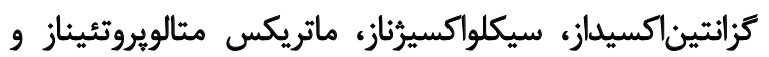

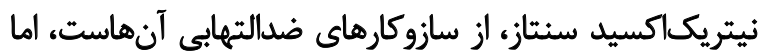

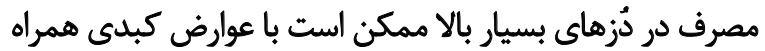

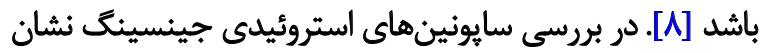

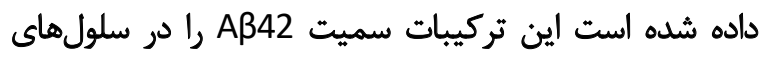

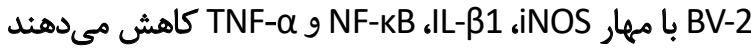

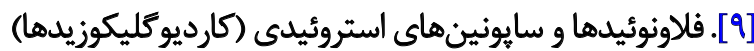

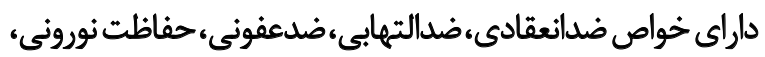

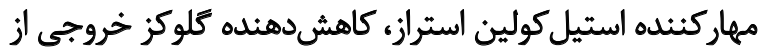

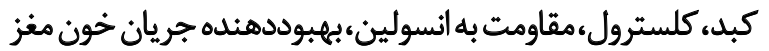

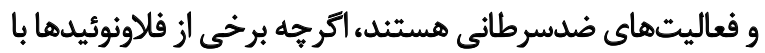

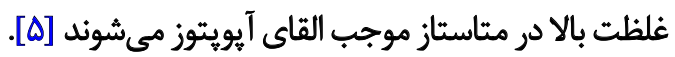
آميلوئيد بتا باعث از دست رفتن فعاليت كولينرزيك از طريق

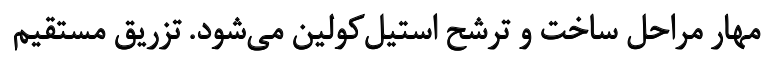

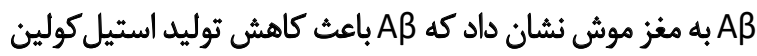

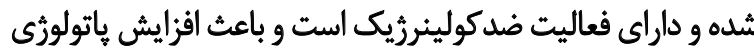

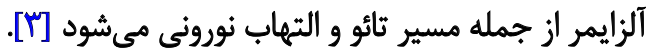

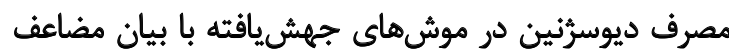

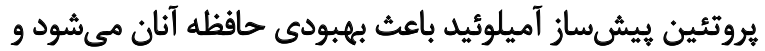

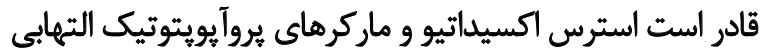

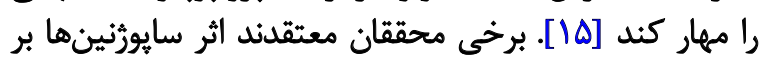

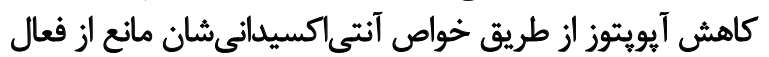

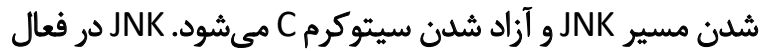

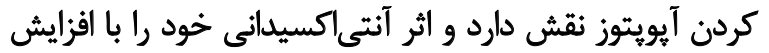

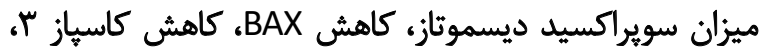
مهار ROS و تنظيم خانواده BCL-Y بروز مي كائهد. اثر مصرف 
استخراج از كياه، توسط شيكر در يك سىسى نرمال سالين كاملاً

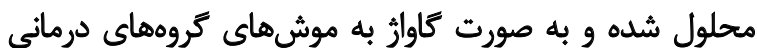
خورائده مى شيد.

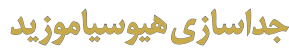

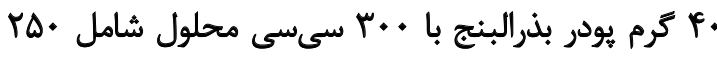

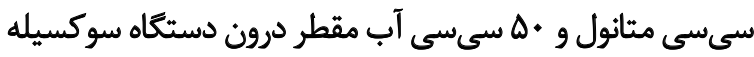

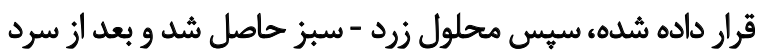

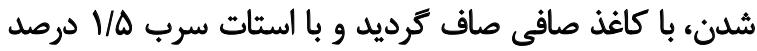

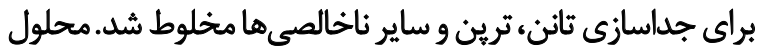

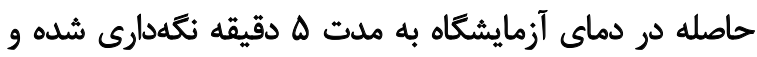

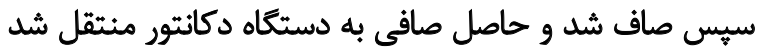

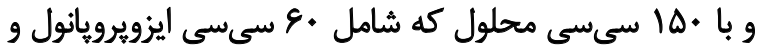

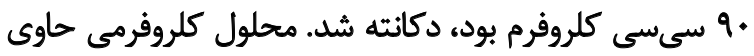

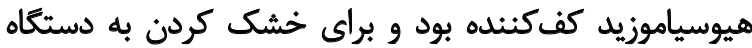

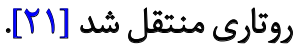

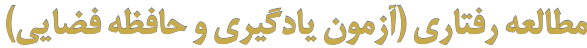

در اين بررسى، سنجش ياديّيرى و حافظه فضايى توسط ماز

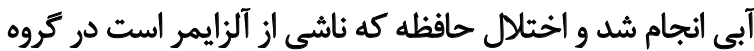

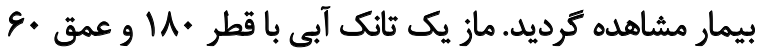

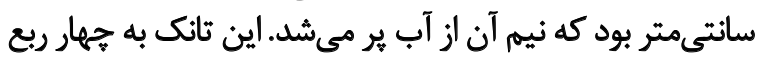

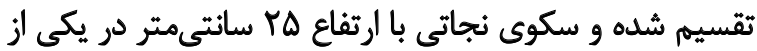

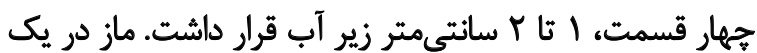

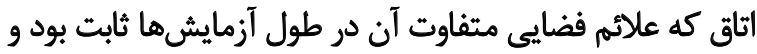

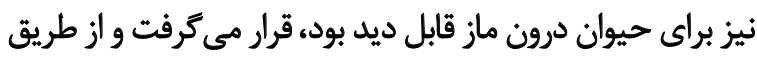

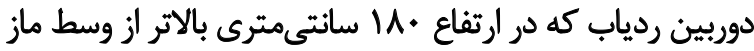

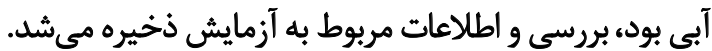

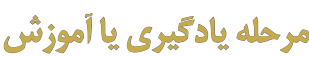

حيوان در طى آزمايش از يكى از ربعهاى ماز به طورى كه

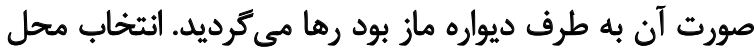

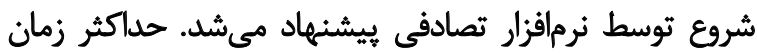

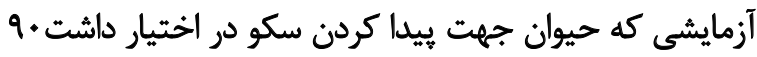

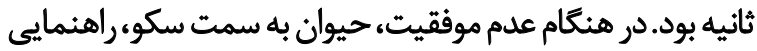

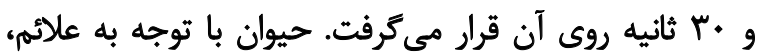

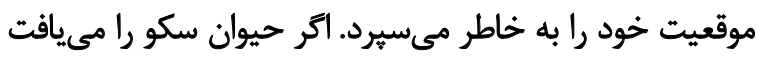

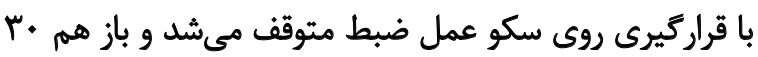

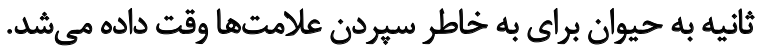

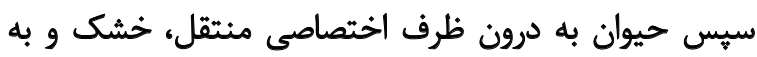

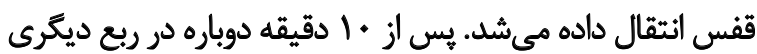

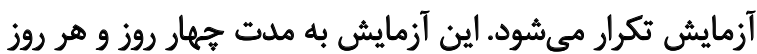
جهار بار تكرار مىشد.

\section{مواد و روشها}

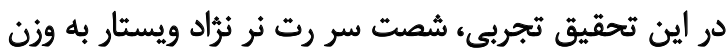

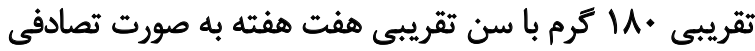

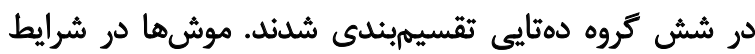

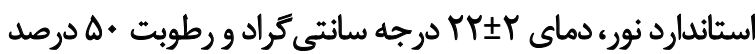

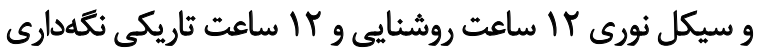

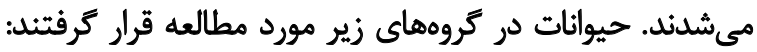

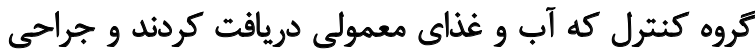

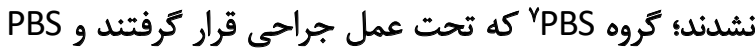

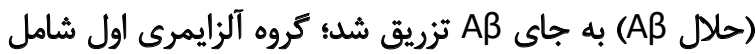

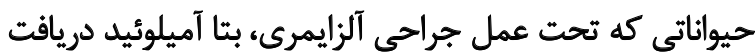

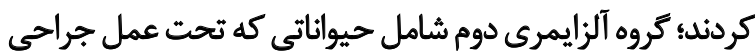

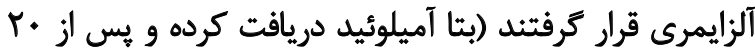

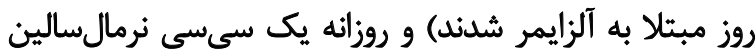

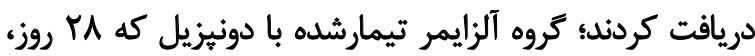

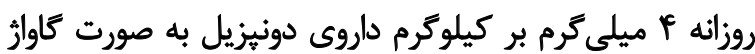

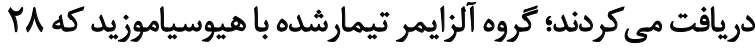

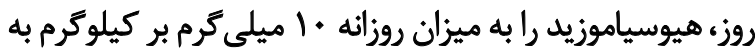

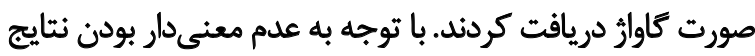

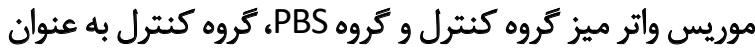

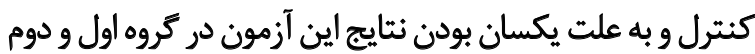

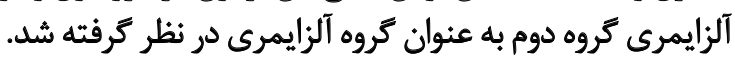
داروى بتا آميلوئيد از شركت Sigma Aldrich به ميزان .

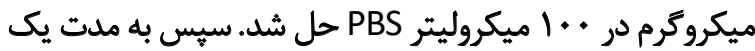

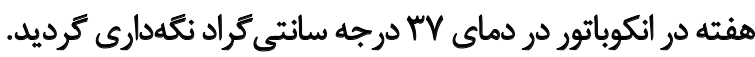
حيوانات باتزريق كتامين (•وميلى كرم بر كيلوكرم) وزايلازين

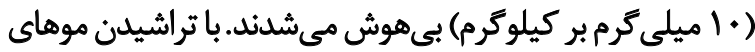

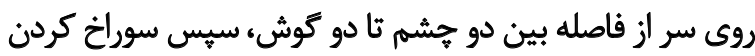

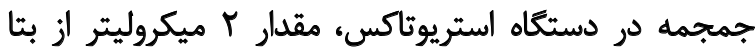

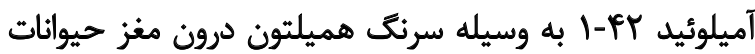

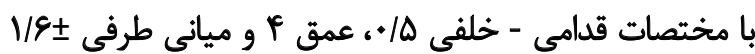

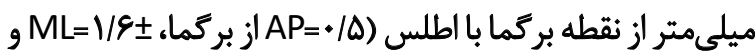

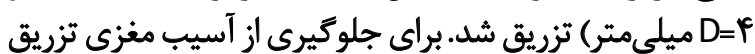

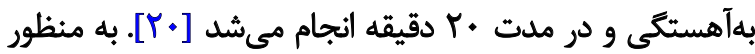

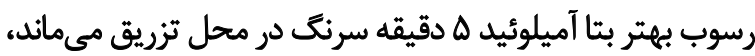

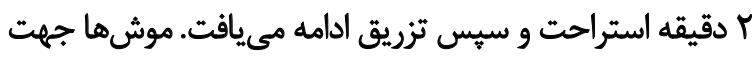

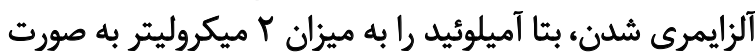

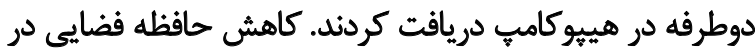

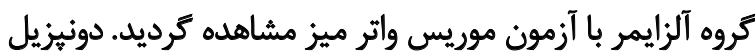

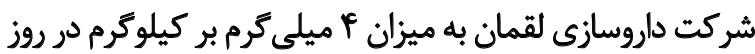

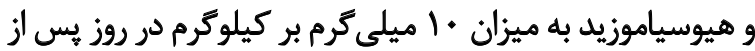

7. Phosphate buffered saline 


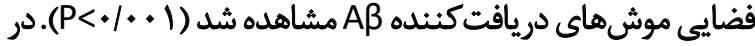

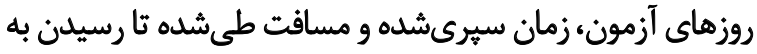

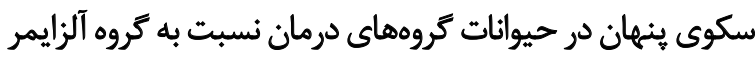
كاهش يافت (تصوير شماره (1).

در آزمون يروب (كه بدون سكوى هينهان اجرا شد)، زمان

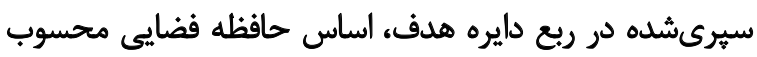

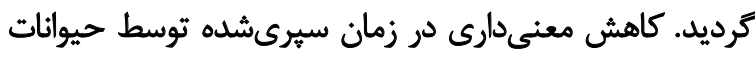

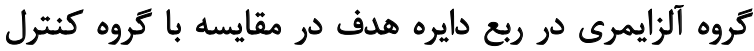

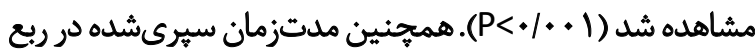

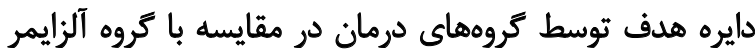

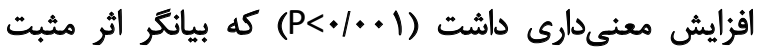

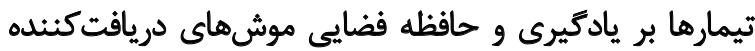

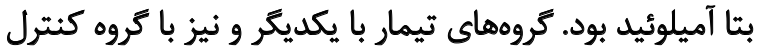

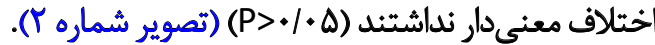

4

در اين مطالعه تجربى، اثر دونيزيل و هيوسياموزيد بر بهبود

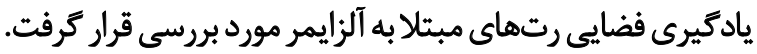

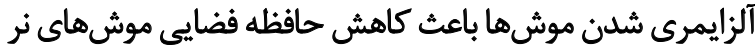

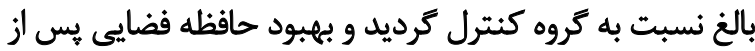

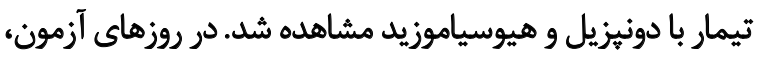

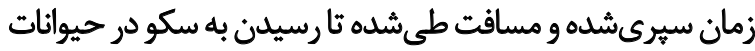

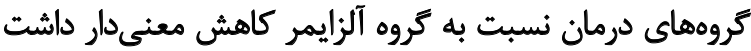

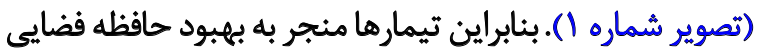

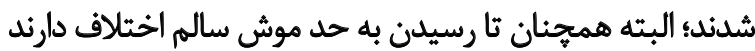

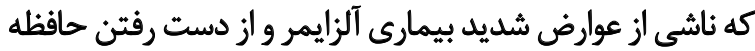

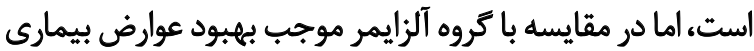

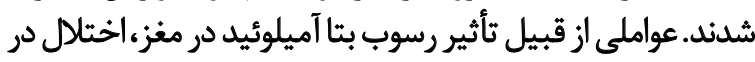

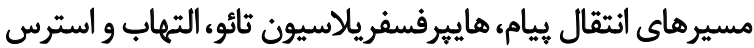

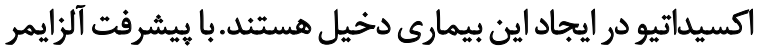

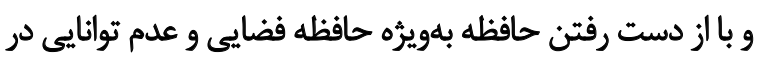

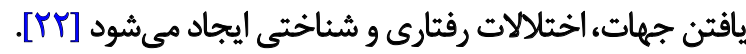

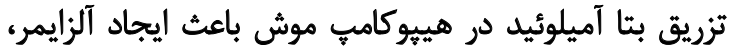

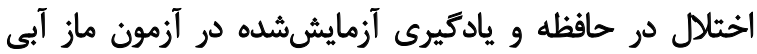

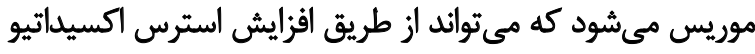

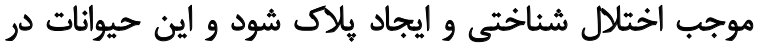

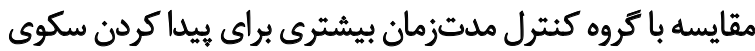

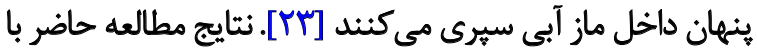

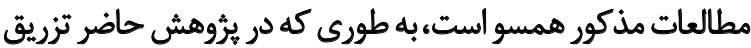

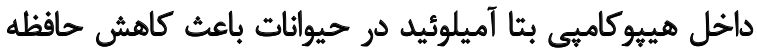

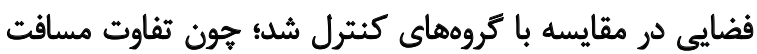

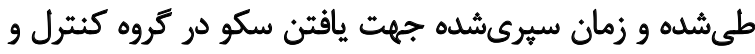

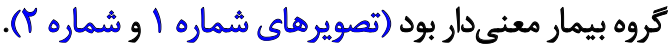

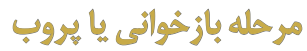

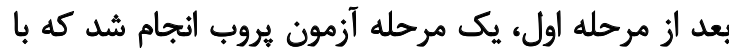

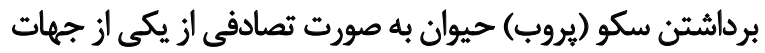

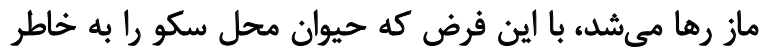

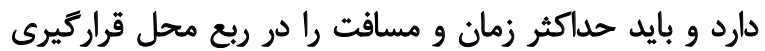

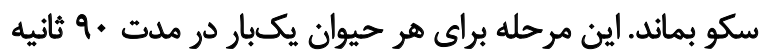

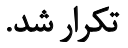

در اين تحقيق از نرمافزار SPSS نسخه با براي تجزيه و تحليل

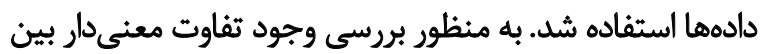

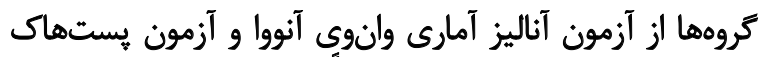

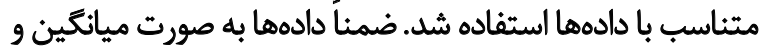

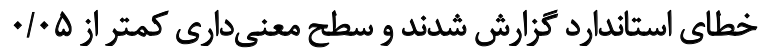

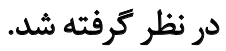

ياقتهاهما

بر اساس نتايج آزمون ماز آبى موريس، يادكيرى و حافظه

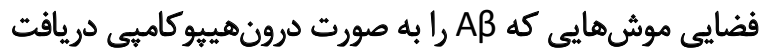

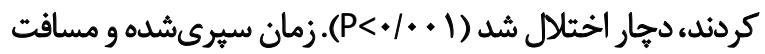

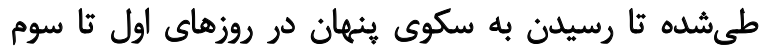

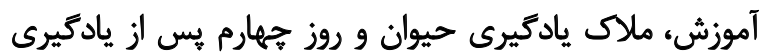

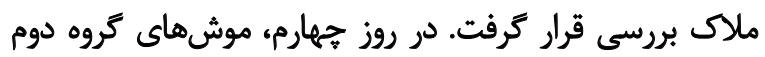

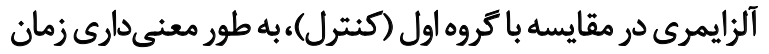

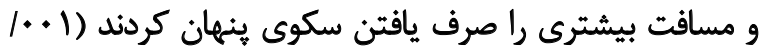

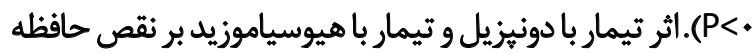

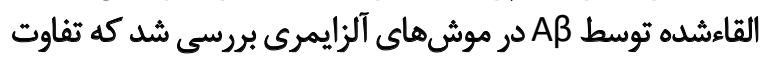

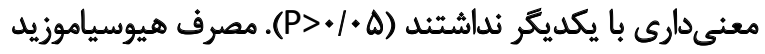

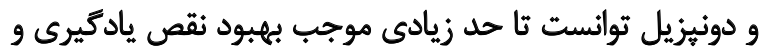

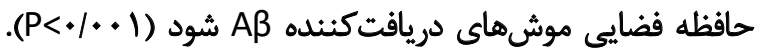

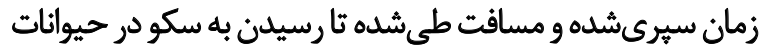

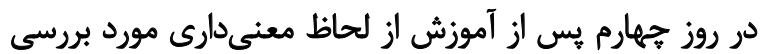

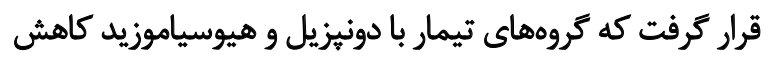

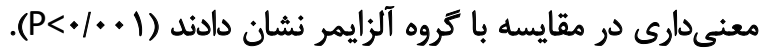

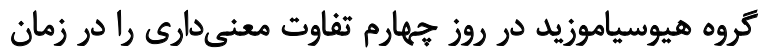

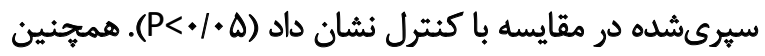

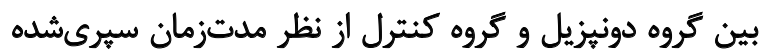

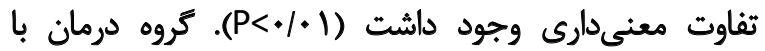

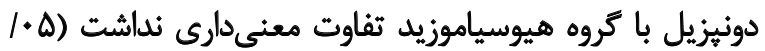
(P>.

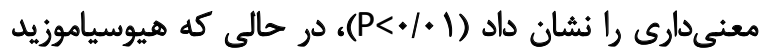

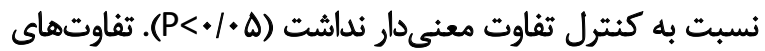

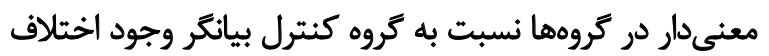

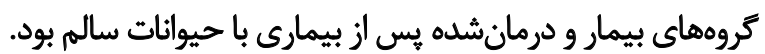

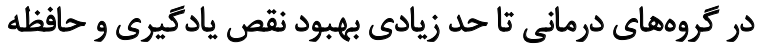




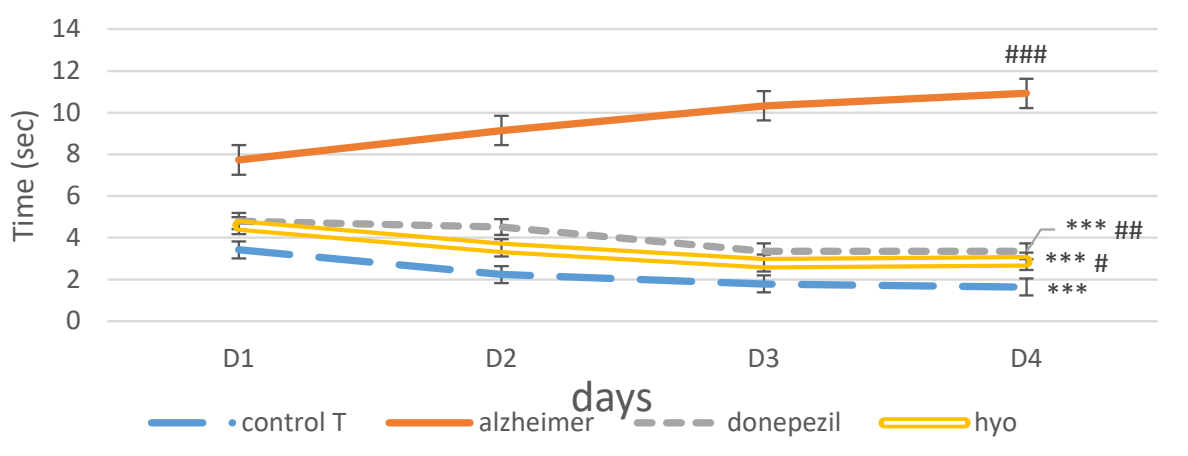

الـن (

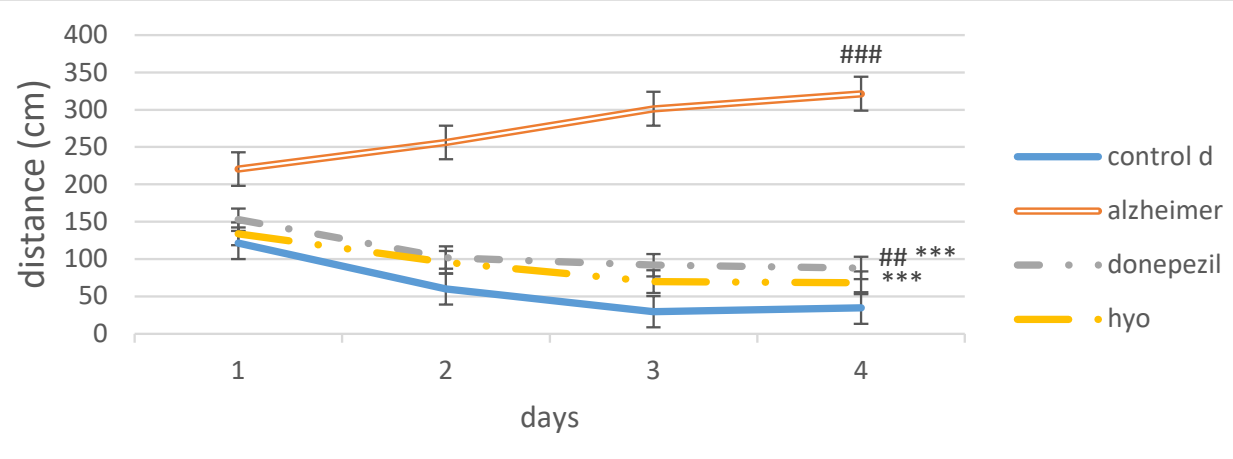

(ب

تصوير ا. بررسى تأثير تيمار با دونيزيل و هيوسياموزيد بر حيوانات آلزايمرى با استفاده از آزمون ماز آبى موريس

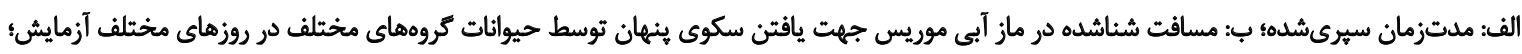

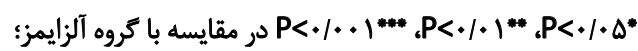

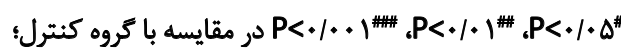
Hyo

مطابق با يافتهاى اين تحقيق،استفاده از سايورذنين استروئيدى

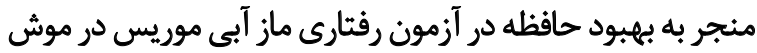

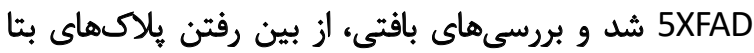

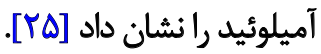
در موش با تجمع بتا آميلوئيد، فعاليت AChE (استيل كولين

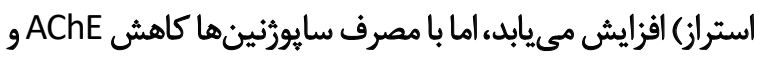

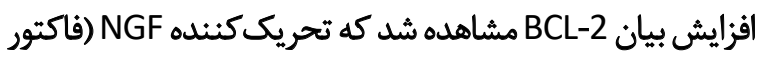

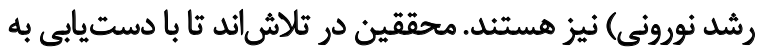

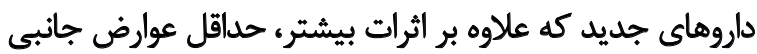

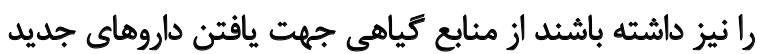

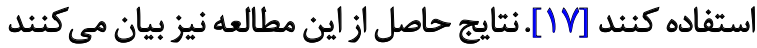

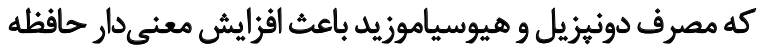
فضايى موشها شده كه همسو با مطالعات كذشته است
موافق با مطالعه حاضر، رسولى جازى و همكاران تزارش دادئ دئد

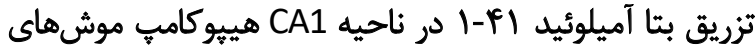

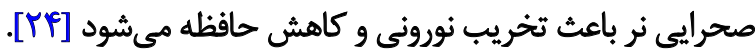

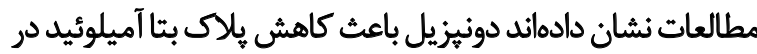

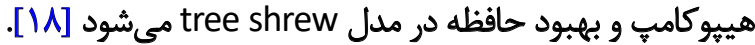

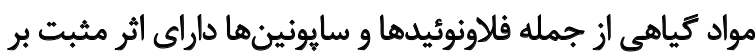

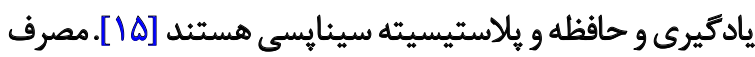

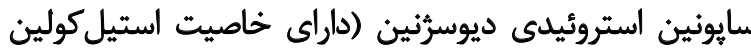

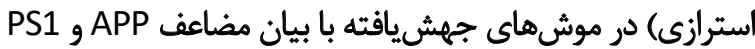

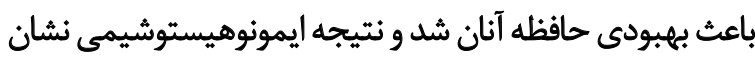

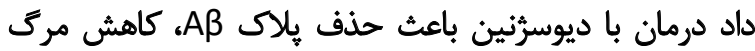

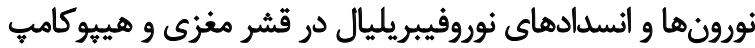

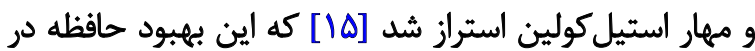
يُوروهش حاضر نيز مشهود بود. 


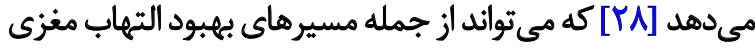

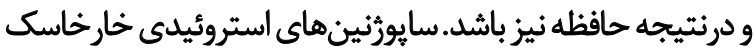

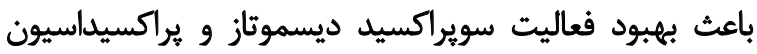

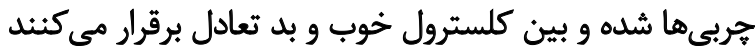

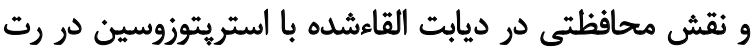

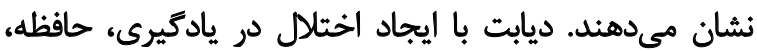

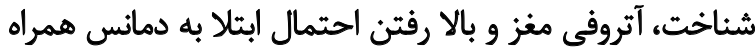

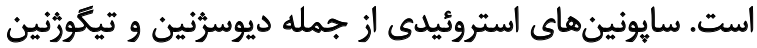

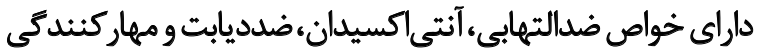

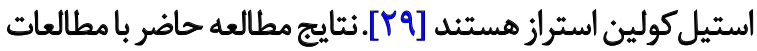

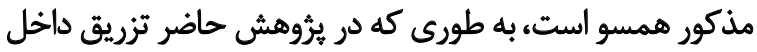

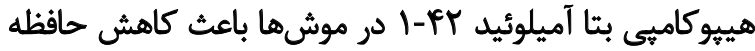

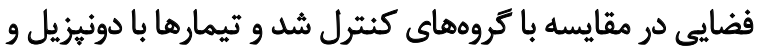

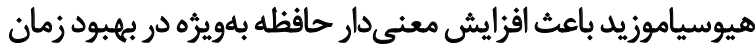

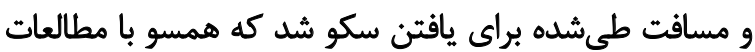

كذُشته است.

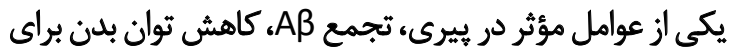

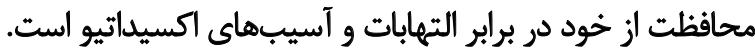

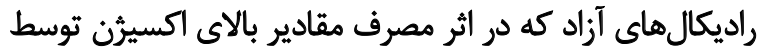

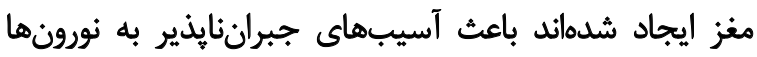

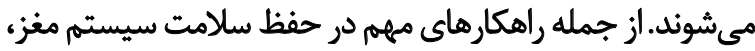

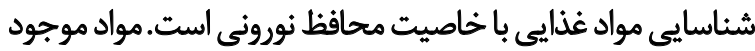

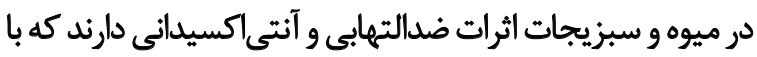

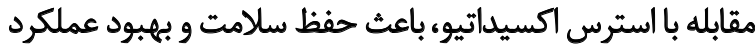

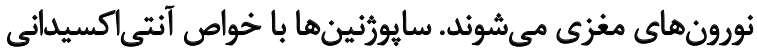

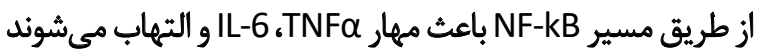

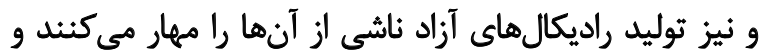

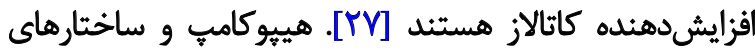

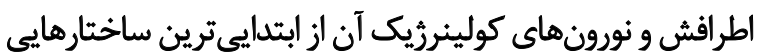

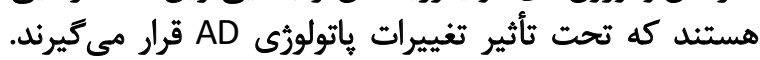

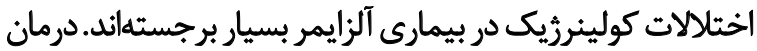

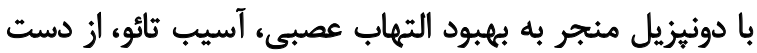

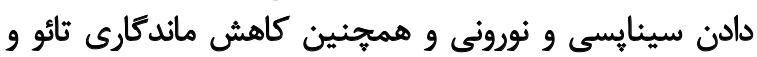

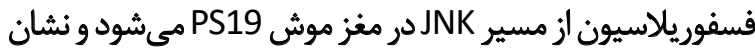

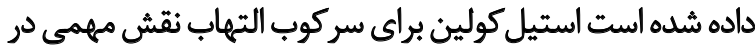

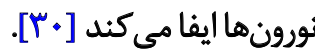

برخلاف نتايج مطالعه حاضر با ايجاد تخريب در حافظه با تزريق

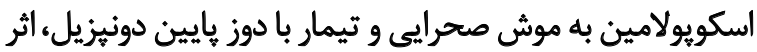

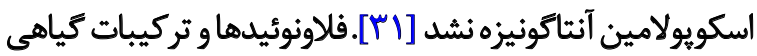

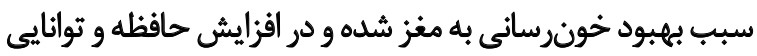

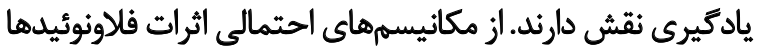

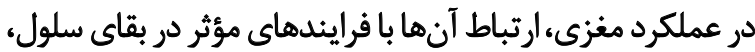

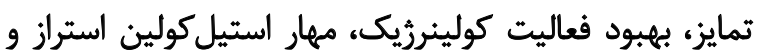

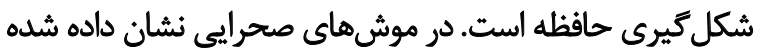

علائم اوليه بيمارى آلزايمر، مختل شدن تدريجى حافظه

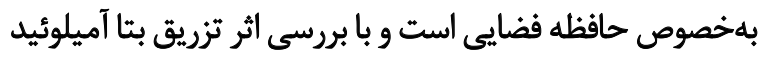

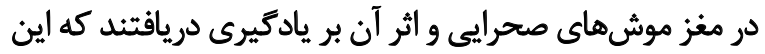

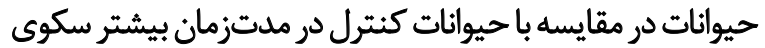

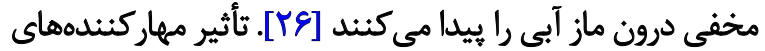

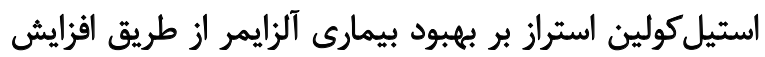

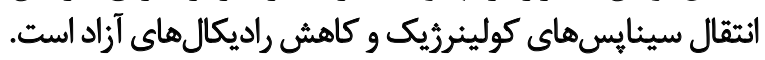

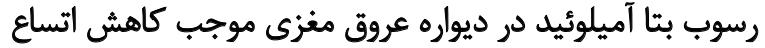

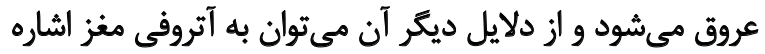

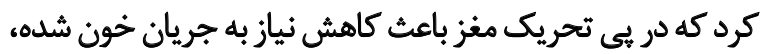

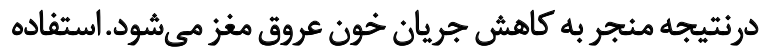

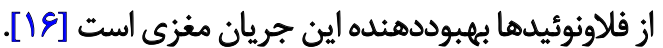

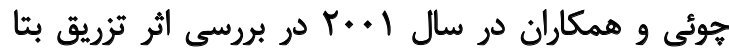

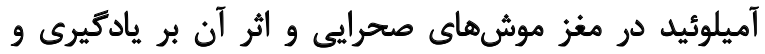

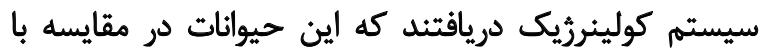

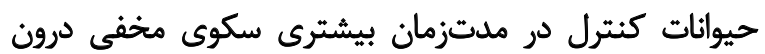

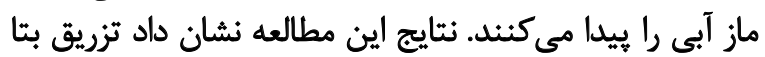

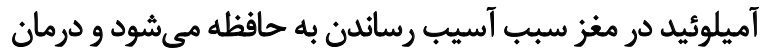

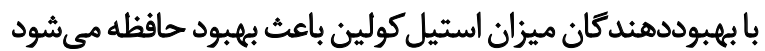

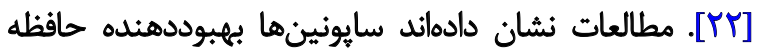

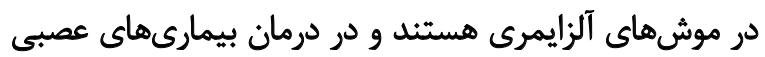

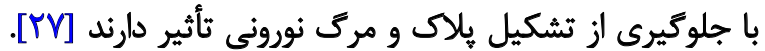

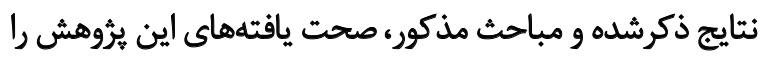

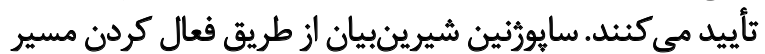

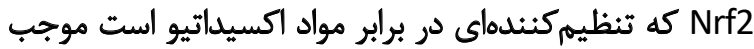

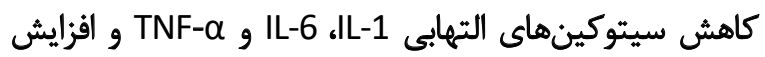

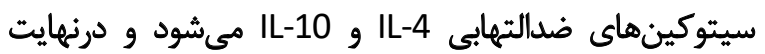

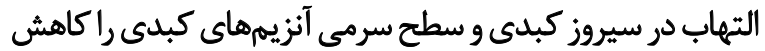
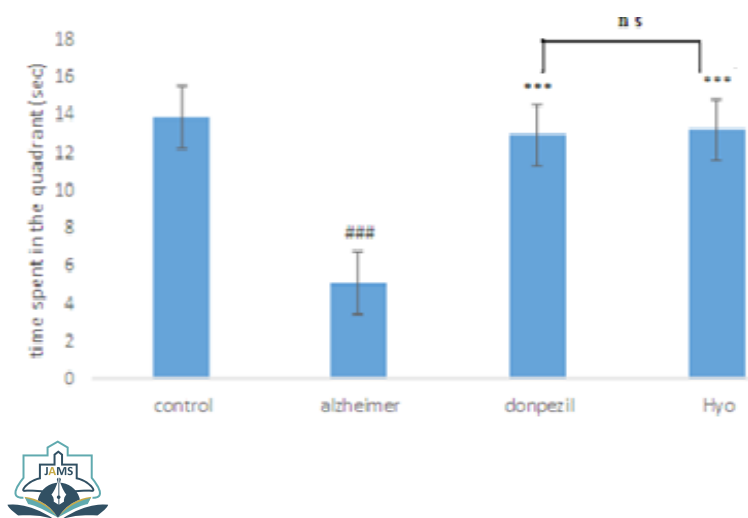

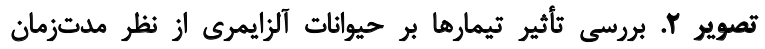

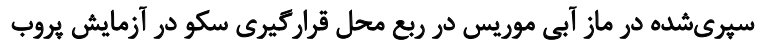

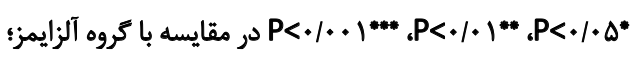

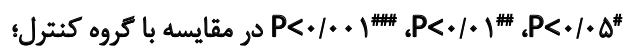
Hyo 
مى دهند AChEl، سلول را به طور مستقيم در برابر آسيب ناشى مئى

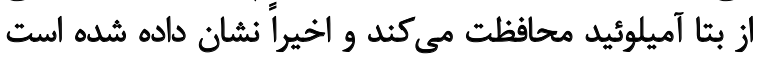

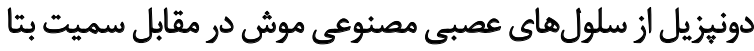

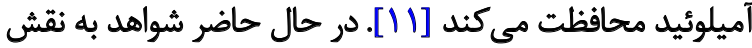

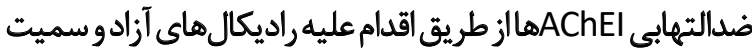

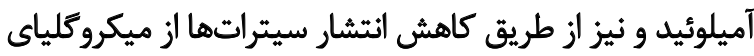

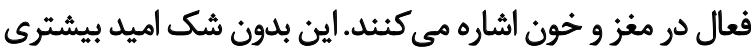

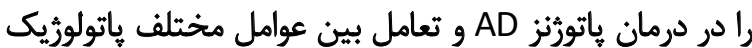

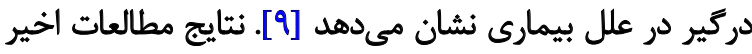

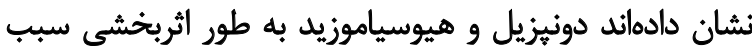

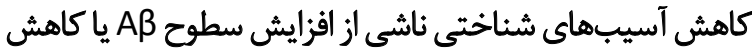

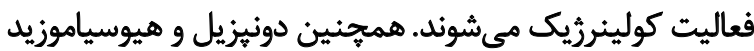

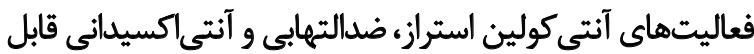
ملاحظهاى راطبق مطالعات انجامشده در ساليان كَذشته اعمال مي كنثل.

اثر دونيزيل از طريق جندين مكانيسم شامل فعاليتهاى إنى

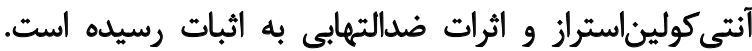

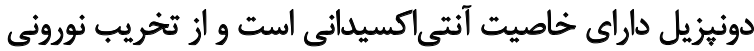

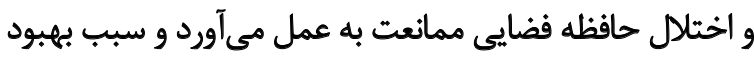

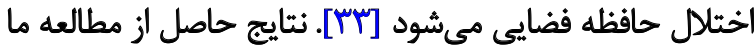

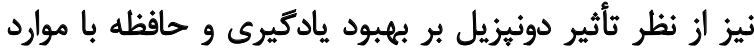

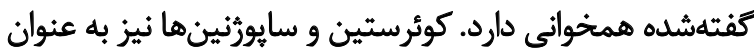

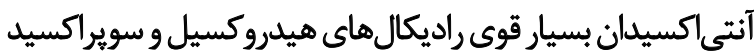

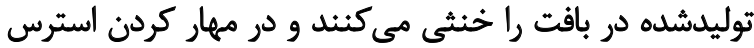

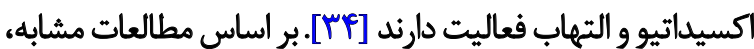

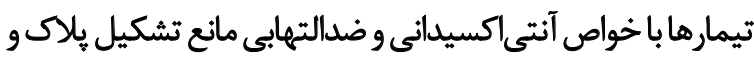

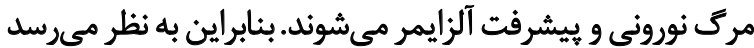

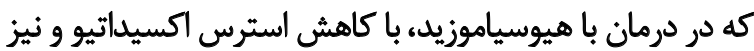

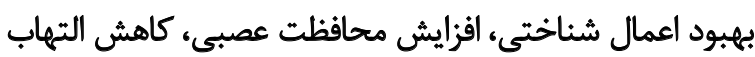

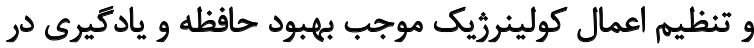

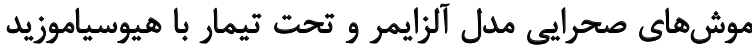

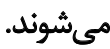

\section{تئيجهاكيرى}

با توجه به نتايج حاصل از مطالعات كذشته و مطالعه اخير

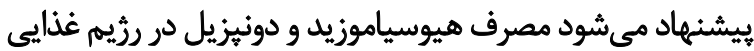

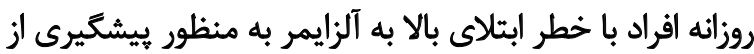

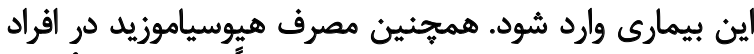

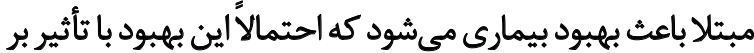

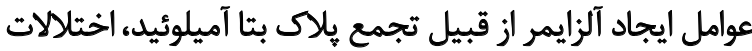

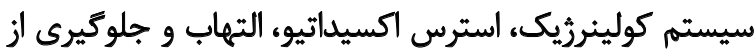
آيويتوز نورونى صورت مى كيرد.
است تركيبهاى فلاونوئيدى قادر به اثركذارى مثبت بر حافظه

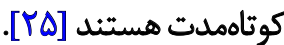
با توجه به نتايج مطالعه حاضر، كاهش زمان سيرىشده

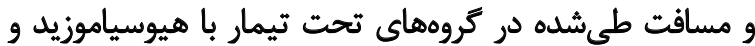

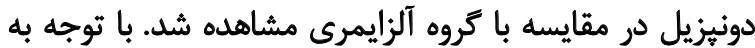

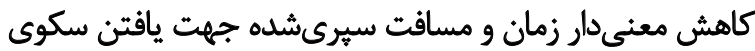

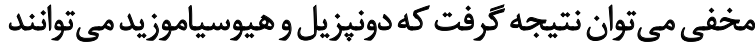

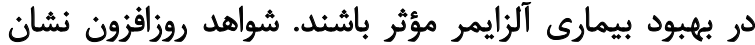

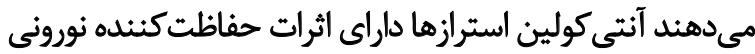

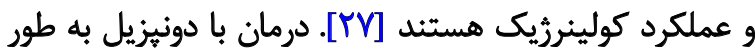

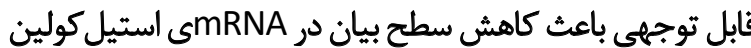

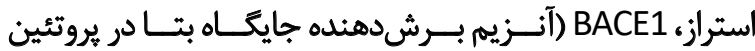

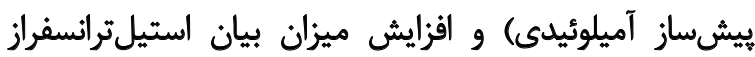

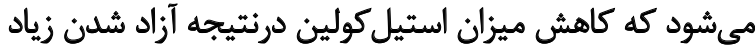

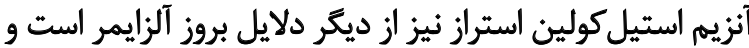

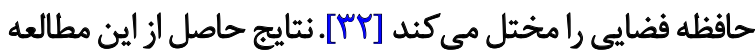

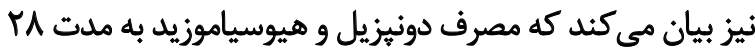

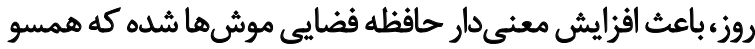
با مطالعات كُشُشته است.

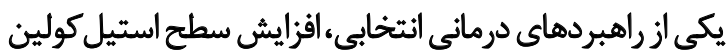

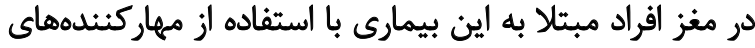

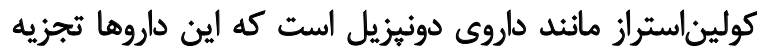

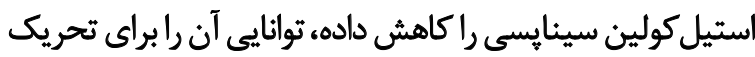

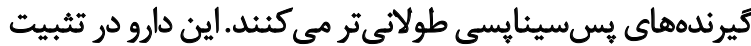

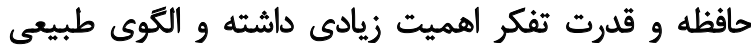

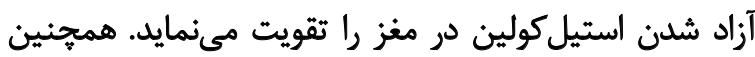

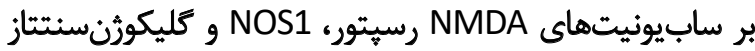

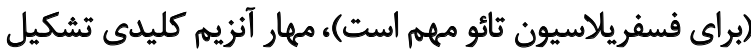

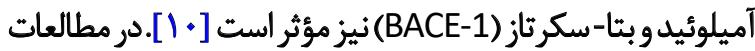

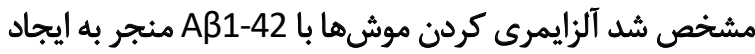

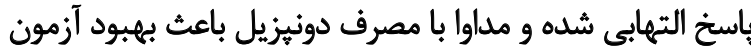

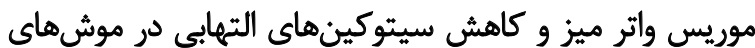

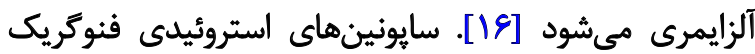

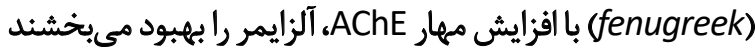
و وييشنهاد شده است كه اين تركيبات با خاصيت آنتى اكسيداني

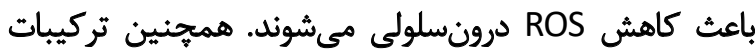

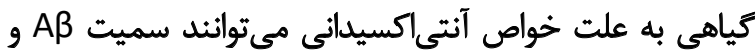

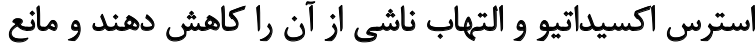

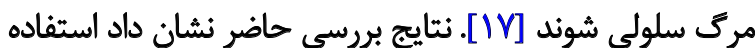

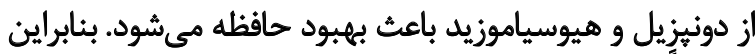

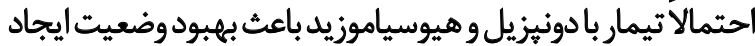

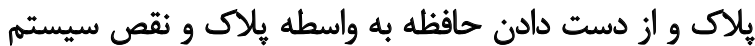

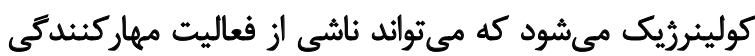

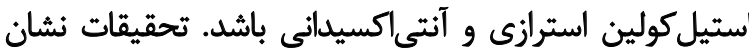




\section{ملاحظات اخلاقى}

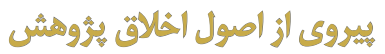

اين تحقيق در دانشكاه آزاد اسلامى، واحد علوم و تحقيقات با شناسه اخلاق REC.1397.057. IR.IAU.SRB تأييد شده است.

$$
\text { o اon }
$$

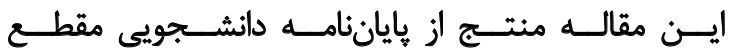

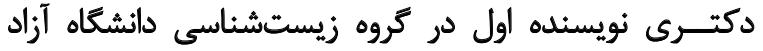

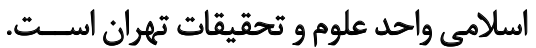

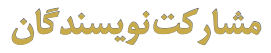

تمامى نويسندكان معيارهاى اسثاندارد نويسندكى بر اساس

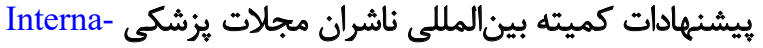
را راري بودند و همكى به يك اندازه در نتارش اثر مشاركت داشتند.

$$
\text { ثقار ضن مثأع }
$$

بدينوسيله نويسندكان تصريح مى كنند كه هيجكونه تضاد منافعى در خصوص يروهش حاضر وجود ندارد. 


\section{References}

[1] Liu C-C, Kanekiyo T, Xu H, Bu G. Apolipoprotein E and Alzheimer disease: Risk, mechanisms and therapy. Nat Rev Neurol. 2013 ; 9(2):106-18. [DOI:10.1038/nrneurol.2012.263] [PMID] [PMCID]

[2] Hebert LE, Beckett LA, Scherr PA, Evans DA. Annual incidence of Alzheimer disease in the United States projected to the years 2000 through 2050. Alzheimer Dis Assoc Disord. 2001; 15(4):169-73. [DOI:10.1097/00002093-200110000-00002] [PMID]

[3] Behl C. Oxidative stress in Alzheimer's disease: Implications for prevention and therapy. Subcell Biochem. 2005; 38:65-78. [DOI:10.1007/0387-23226-5_3] [PMID]

[4] Profenno LA, Porsteinsson AP, Faraone SV. Metaanalysis of Alzheimer's disease risk with obesity, diabetes, and related disorders. Biol Psychiatry. 2010; 67(6):505-12 [DOI:10.1016/j.biopsych.2009.02.013] [PMID]

[5] Chiu F-L, Lin J-K. Tomatidine inhibits iNOS and COX-2 through suppression of NF-KB and JNK pathways in LPS-stimulated mouse macrophages. FEBS Lett. 2008; 582(16):2407-12. [DOI:10.1016/j.febslet.2008.05.049] [PMID]

[6] Lunga I, Chintea P, Piacente S, Şveţ S, Bassarello C, Pizza C. Steroida saponins from the seeds of Hyoscyamus niger L. Chem J Moldova. 2008; 3(1):89-93. https://ibn.idsi.md/ro/vizualizare_articol/6834

[7] Reisberg B, Doody R, Stoffler A, Schmitt F, Ferris S, Mobius HJ. Memantine in moderate-to-severe Alzheimer's disease. N Engl J Med. 2003; 348(14):1333-4. [DOI:10.1056/NEJMoa013128] [PMID]

[8] Hur J, Lee P, Moon E, Kang I, Kim S-H, Oh MS, et al. Neurite outgrowth induced by spicatoside $A$, a steroidal saponin, via the tyrosine kinase $A$ receptor pathway. Eur J Pharmacol. 2009; 620(1-3):9-15. [DOI:10.1016/j. ejphar.2009.08.016] [PMID]

[9] Koh E-K, Yun W-B, Kim J-E, Song S-H, Sung J-E, Lee H-A, et al. Beneficial effect of diosgenin as a stimulator of NGF on the brain with neuronal damage induced by $A \beta-42$ accumulation and neurotoxicant injection. Lab Anim Res. 2016; 32(2):105-15. [DOI:10.5625/lar.2016.32.2.105] [PMID] [PMCID]

[10] Black SE, Doody R, Li H, McRae T, Jambor KM, Xu Y, et al. Donepezil preserves cognition and global function in patients with severe Alzheimer disease. Neurology. 2007; 69(5):459-69. [DOI:10.1212/01. wnl.0000266627.96040.5a] [PMID]

[11] Yoshiyama Y, Kojima A, Ishikawa C, Arai K. Anti-inflammatory action of donepezil ameliorates tau pathology, synaptic loss, and neurodegeneration in a tauopathy mouse model . J Alzheimers Dis. 2010; 22(1):295306. [DOI:10.3233/JAD-2010-100681] [PMID]

[12] Selkoe DJ, Hardy J. The amyloid hypothesis of Alzheimer's disease at 25 years. EMBO Mol Med. 2016; 8(6):595-608. [DOI:10.15252/ emmm.201606210] [PMID] [PMCID]

[13] Jang K-J, Kim HK, Han MH, Oh YN, Yoon H-M, Chung YH, et al. Antiinflmmatory effects of saponins derived from the roots of Platycodon grandiflrus in lipopolysaccharide-stimulated BV2 microglial cells. Int J Mol Med. 2013; 31(6):1357-66. [DOI:10.3892/ijmm.2013.1330] [PMID]

[14] Fischer CW, Elfving B, Lund S, Wegener G. Behavioral and systemic consequences of long-term inflmmatory challenge. J Neuroimmunol. 2015; 288:40-6. [DOI:10.1016/j.jneuroim.2015.08.011] [PMID]

[15] Tohda C, Urano T, Umezaki M, Nemere I, Kuboyama T. Diosgenin is an exogenous activator of 1,25D3-MARRS/Pdia3/ ERp57 and improves Alzheimer's disease pathologies in 5XFAD mice. Sci Rep. 2012; 2:535. [DOI:10.1038/srep00535] [PMID] [PMCID]
[16] Ji KY, Kim KM, Kim YH, Im A-R, Lee JY, Park B, et al. The enhancing immune response and anti-inflammatory effects of Anemarrhena asphodeloides extract in RAW 264.7 cells. Phytomedicine. 2019; 59:152789. [DOI:10.1016/j.phymed.2018.12.012] [PMID]

[17] Khalil WKB, Roshdy HM, Kassem SM. The potential therapeutic role of Fenugreek saponin against Alzheimer's disease: Evaluation of apoptotic and acetylcholinesterase inhibitory activities. J Appl Pharm Sci. 2016; 6(9):166-73. [DOI:10.7324/JAPS.2016.60925]

[18] Huang S-H, Wang J, Sui W-H, Chen B, Zhang X-Y, Yan J, et al. BDNFdependent recycling facilitates TrkB translocation to postsynaptic density during LTP via a Rab11-dependent pathway. J Neurosci. 2013; 33(21):9214-30 [DOI:10.1523/JNEUROSCI.3256-12.2013] [PMID] [PMCID]

[19] Solfrizzi V, Panza F, Frisardi V, Seripa D, Logroscino G, Imbimbo $\mathrm{BP}$, et al. Diet and Alzheimer's disease risk factors or prevention: The current evidence. Expert Rev Neurother. 2011; 11(5):677708. [DOI:10.1586/ern.11.56] [PMID]

[20] Ghahremanitamadon F, Shahidi S, Zargooshnia S, Nikkhah A Ranjbar A, Soleimani Asl S. Protective effects of borago officinalis extract on amyloid. Biomed Res Int. 2014; 2014:798535. [DOI:10.1155/2014/798535] [PMID] [PMCID]

[21] Dhawan D, Gupta J. Comparison of different solvents for phytochemical extraction potential from Datura metel plant leaves. Int J Biol Chem. 2017; 11(1):17-22. [DOI:10.3923/ijbc.2017.17.22]

[22] Choi SH, Park CH, Koo JW, Seo JH, Kim HS, Jeong SJ, et al. Memory impairment and cholinergic dysfunction by centrally administered $A \beta$ and carboxyl-terminal fragment of Alzheimer's APP in mice. FASEB J. 2001; 15(10):1816-8. [DOI:10.1096/fj.00-0859fje] [PMID]

[23] Guariglia CC. Spatial working memory in Alzheimer's disease. Dement Neuropsychol. 2007; 1(4):392-5. [DOI:10.1590/S198057642008DN10400011] [PMID] [PMCID]

[24] Rasoolijazi H, Azad N, Joghataei MT, Kerdari M, Nikbakht F Soleimani M. The protective role of carnosic acid against betaamyloid toxicity in rats. Scient World J. 2013; 2013:917082. [DOI:10.1155/2013/917082] [PMID] [PMCID]

[25] Tabet N. Acetylcholinesterase inhibitors for Alzheimer's disease: Anti-inflmmatories in acetylcholine clothing. Age Age. 2006; 35(4):336-8. https://doi.org/10.1093/ageing/afl027

[26] Buee L, Bussiere T, Buee-Scherrer V, Delacourte A, Hof PR. Tau protein isoforms, phosphorylation and role in neurodegenerative disorders. Brain Res Brain Res Rev. 2000; 33(1):95-130. [DOI. org/10.1016/S0165-0173(00)00019-9]

[27] Shal B, Ding W, Ali H, Kim YS, Khan S. Anti-neuroinflmmatory potential of natural products in attenuation of Alzheimer's disease. Front Pharmacol 2018; 9:548-64. [DOI.org/10.3389/ fphar.2018.00548]

[28] Roghani M, Baluchnejad Mojarrad T, Andalibi N, Ansari F, Sharayeli M. [Effect of consumption of T. terrestris on serum glucose and lipid levels in diabetic rats (Persian)]. JSSU. 2010; 18(1):17-23. http://jssu.ssu.ac.ir/article-1-1005-en.html

[29] Gong G, Qin Y, Huang W, Zhou S, Wu X, Yang X, et al. Protective effects of diosgenin in the hyperlipidemic rat model and in human vascular endothelial cells against hydrogen peroxide-induced apoptosis. Chem Biol Interact. 2010; 184(3):366-75. [DOI:10.1016/j cbi.2010.02.005] [PMID] 
[30] Ramos-Rodriguez JJ, Pacheco-Herrero M, Thyssen D, MurilloCarretero MI, Berrocoso E, et al. Rapid beta-amyloid deposition and cognitive impairment after cholinergic denervation in APP/PS1 mice. J Neuropathol Exp Neurol. 2013; 72(4):272-85 [DOI:10.1097/ NEN.0b013e318288a8dd] [PMID] [PMCID]

[31] Eskandary A, Moazedi AA. [Effect of co-administration of donepezil and folic acid on spatial memory impairment in adult male rat model of Alzheimer's disease (Persian)]. JQUMS.2018; 22(5):14-25. [DOI:10.29252/qums.22.5.14]

[32] Birch AM, Katsouri L, Sastre M. Modulation of inflammation in transgenic models of Alzheimer's disease. J Neuroinflammation. 2014; 11:25. [DOI:10.1186/1742-2094-11-25] [PMID] [PMCID]

[33] Umukoro S, Adewole FA, Eduviere AT, Aderibigbe AO, Onwuchekwa C. Free radical scavenging effect of donepezil as the possible contribution to its memory enhancing activity in mice. Drug Res (Stuttg). 2014; 64(5):236-9. [DOI:10.1055/s-0033-1357126] [PMID]

[34] Chen Y, Miao Y, Huang L, Li J, Sun H, Zhao Y, et al. Antioxidant activities of saponins extracted from Radix Trichosanthis: An in vivo and in vitro evaluation. BMC Complement Altern Med. 2014; 14:86. [DOI:10.1186/1472-6882-14-86] [PMID] [PMCID] 